\title{
MÉTA-MÉDIATIONS COOPÉRATIVES EN SÉMANTIQUE DES TERMES : UN SYSTÈME D'ÉTUDE ET DE PRÉSENTATION DES SENS D'ITEMS LEXICAUX DANS DIVERS DOMAINES D'OCCURRENCE
}

\author{
Michele DE GIOIA \\ Università degli Studi di Padova \\ Ioannis KANELLOS \\ IMT Atlantique
}

\begin{abstract}
En): In this paper we present the results of the "Onto-terminology of mediation" research project. The project aims at introducing a methodology, a system and, above all, a particular cooperative mode in coping with some terminological issues, in particular, the comparative exploration of the sense of a term in various domains. The use-case concerns the term "mediation". We have chosen 7 terms addressed by mediation, occurring in five different domains: law, education, communication, language and culture. The suggested approach finds roots in a corpus-based linguistic paradigm. It intends to build up a cooperative platform offering assistance to users preoccupied with the semantics of a term, while that term is used in one of these domains. More concretely, we propose: i. a way of modeling the terminological data, typically extracted from text corpora, ii. an organization of these data into particular knowledge bases and iii. a cooperative manner of presenting them critically. Such presentations may take various forms, the most prominent being the pedagogical one, consisting in producing reasoned discourses on term semantics. In the first part of the paper we discuss not only the interest of the project and its horizon, but also its limits. In the second part, we explain the underlying methodology and outline some possible cooperative semantic scenarios. Finally, in the third part, we describe the use of the developed system-through some examples. We conclude with a series of considerations on the transmission and sharing of terminological knowledge, emerging from and shaped by such a cooperative semantic mode.
\end{abstract}

Keywords (En): comparative terminology; descriptive terminology; cooperative terminology; domain semantics; mediation; corpus-based linguistics; intertextuality.

Résumé (Fr) : Dans cet article, nous présentons les résultats du projet de recherche «Ontoterminologie de la médiation ». Il s'agit d'un projet qui vise à promulguer une méthodologie, un système, mais surtout un mode de travail coopératif pour aborder divers problèmes liés à l'exploration trans-domaniale du sens d'un terme - en l'occurrence, celui de " médiation ». Nous avons choisi 7 termes relevant du concept de médiation, tels qu'ils apparaissent dans cinq domaines différents : ceux du droit, de la formation, de la communication, du langage et de la culture. L'approche préconisée se fonde sur le paradigme d'une linguistique de corpus. Elle entend mettre sur pied une plateforme de coopération au service d'utilisateurs concernés par la sémantique des termes d'un domaine particulier. Concrètement, il s'agit : i. de modéliser certaines données sémantiques, issues typiquement de corpora textuels, ii. de les consigner, ensuite, dans des bases de connaissances appropriées et iii. d'offrir, enfin, la possibilité de les faire présenter de manière critique et collaborative. De telles présentations peuvent prendre diverses formes, dont une, pédagogique, consiste en la possibilité de construire des discours raisonnés sur la sémantique des termes. Dans la première partie de l'article, nous discutons de l'intérêt d'un tel projet, de son horizon, mais aussi de ses limites. Dans la seconde, nous explicitons la méthodologie et précisons les scénarios de coopération sémantique possibles. Dans la troisième, enfin, nous décrivons l'utilisation du système mis en place, par une série d'exemples. Nous concluons avec une série de réflexions concernant la transmission et le partage des connaissances émergeant $d e$ et se formatant par ce mode de coopération sémantique. 
Mots-clés (Fr): terminologie comparative; terminologie descriptive ; terminologie collaborative ; sémantique de domaine ; médiation ; linguistique de corpus ; intertextualité.

\section{Introduction}

Nous présentons dans cet article les premiers résultats du projet de recherche «Onto-terminologie de la médiation». Ce projet doit ses origines à un constat empirique : l'extraordinaire fortune, de nos jours, du terme « médiation » ainsi que de sa pénétration dans un nombre significatif de domaines, de pratiques, de discours, d'activités et d'usages. Il s'agit de trouver des moyens techniques efficaces pour suivre les mutations sémantiques du terme en suivant les traces qu'elles laissent dans les textes (BOURIGAULT \& SLODZIAN, 1999, L'HOMME, 2004, RASTIER, 2009, ISO 704 2009, entre bien d'autres). Le projet s'inscrit dans la continuité des recherches menées, depuis 2010, au Département SPGI (des sciences politiques, juridiques et des études internationales) de l'Université de Padoue (DE GIOIA, 2019), une activité dont l'ouvrage Mots de médiation. Un lexique bilingue françaisitalien (DE GIOIA \& MARCON, 2014), constitue l'acte de naissance. Cet ouvrage vise à tracer le champ lexical de la médiation comme champ multi-domanial et polymorphique, dégagé de la culture contentieuse, traditionnellement de facture juridique.

On le sait, l'appropriation d'un terme au sein d'une pratique spécifique finit toujours par rénover sa charge sémantique originelle, en lui conférant des sens parfois fort éloignés de son écologie sémantique usuelle : la socialisation du terme induit des flexions sémantiques parfois même imprévisibles (GAUDIN, 2005, TEMMERMAN, 2000). Force est de constater qu'on assiste, aujourd'hui, à une irréfrénable prolifération des significations, des connotations, des acceptions ou des emplois du terme "médiation ». Des définitions alternatives en découlent, faisant de ce terme plus un lieu de problèmes de compréhension et une occasion de mésinterprétations qu'un réel lien d'échanges ; surtout dans le cadre de coopérations interdisciplinaires. En effet, les différentes compréhensions du terme "médiation » témoignent d'un morcellement de son unité conceptuelle ou, pire, d'une sorte de limitation. Cette limitation proviendrait, probablement, de deux tendances :

Premièrement, d'un legs historique: la médiation, dans son acceptation stéréotypée, se veut plutôt une méthode, une technique et un acte de résolution des différends, voire des conflits, empruntant des parcours extrajudiciaires, comme l'arbitrage, la conciliation, la négociation, etc. (LEMPEREUR et al., 2008, GUILLAUME-HOFNUNG, 2002, YARN, 1999).

Deuxièmement, d'une volonté d'opérationnalisation du terme : la limitation correspond à un souci méthodologique de simplification, et se veut, généralement, vecteur d'efficacité dans des pratiques cadrées (BEN MRAD et al., 2008, DE GIOIA \& MARCON, 2020).

On constate ainsi que, dans les divers domaines d'utilisation du concept de médiation, méthodes et techniques associées s'avèrent impossibles à unifier. Les termes fondamentaux, qui composent son champ sémantique, tels que « différend », " conflit», « médiateur», " accord», « impartialité », " indépendance », "processus de médiation", "résolution », etc., désignent ci et là des réalités souvent différentes. Même la relation ternaire d'une médiation, généralement 
omniprésente, se voit subir des déplacements, tant les participants peuvent revêtir des rôles différents et s'inscrire dans des objectifs divers.

Un tel travail, méthodique, devenait critique et semblait s'imposer. Certaines monographies ont tenté de délimiter conceptuellement la médiation et constituent à présent des références multidisciplinaires fiables, au moins dans l'espace francophone. Elles ont posé les bases de l'approche par domaines que nous préconisons dans la suite (section suivante). À titre d'introduction :

1. Pour le domaine du droit: Guillaume-Hofnung (2015), SiX (2001, 2002), GRECO MORASSO (2011), BHATIA et al. (2010), YARN (1999), etc.

2. Pour le domaine de la formation: Coste \& CAVAlli (2015), voir le Vocabulaire de la médiation professionnelle (https://goo.gl/QWV41j), etc.

3. Pour le domaine de la communication : CAUNE (2006), LAFORTUNE (2012), Ben Mrad (2018), MERZEAU (2012), etc.

4. Pour le domaine du langage : DelamotTe (2004, deux volumes), GAVIOLI (2009), GELLEREAU (2011), etc.

5. Pour le domaine de la culture : GAViOLi (2009), CAUne $(1999,2013)$, LAMIZET (1999), VIDAL (2003), etc.

L'appareil théorique italien est beaucoup plus réduit : à l'exception de certains titres (cf. CASTELLI, 1996 et FIORUCCI, 2000), il semble privilégier plutôt la dimension applicative et lucrative.

Toutefois, les différentes contributions scientifiques lors des conférences organisées à l'Université de Padoue et les publications scientifiques qui en ont découlé (DE Giola, 2013 ; De Giola, 2014 ; De Giola et al., 2016 ; DE GiOIA \& MARCON, 2016 ; DE GIOIA et al., 2017) ont mis en évidence l'omniprésence du terme « médiation » dans des discours fort éloignés du droit. Elles ont surtout mis en exergue des conceptualisations aussi riches et importantes que divergentes, en tout cas difficilement compatibles avec le concept de médiation originellement élaboré dans son domaine juridique.

Il s'est ainsi avéré que le domaine juridique ne saurait plus conserver ses droits de préemption sémantique sur le terme. Certes, on retrouve de façon systématique des points de recoupement autour d'une médiation qui serait un processus de communication visant à créer ou à rétablir des liens (GUILLAUME-HOFNUNG, 2015) ; les corpora exploités, tant en français qu'en italien, semblent en attester. Mais, en parallèle, des études linguistiques ont mis en évidence des appropriations qui montrent qu'il y a eu adaptation de la médiation i. à des systèmes conceptuels, ii. à des conditions matérielles, technologiques, sociales, communicationnelles, etc. et iii. à des finalités servant des pratiques idiosyncratiques. C'était bien sûr attendu : on le sait, la praxis impose toujours des déplacements et des affinements sémantiques. Amenant des modifications à son unité conceptuelle originelle, cet état de choses a ainsi impliqué une réduction de son efficacité sociale, corollaire d'une polysémie acquise. Mais aussi, une augmentation du risque d'improductivité dans les interactions interdisciplinaires. 
Devant un tel constat, il y aurait, vraisemblablement, à adopter deux démarches possibles :

I. La première serait de se forger une sorte de "socle terminologique minimal», retraçant, en quelque sorte, l'essentialité du concept de médiation. L'argument serait que, malgré la variation des sens du terme, il persiste un «air de famille », ne serait-ce que par le fait que la médiation convoque toujours une relation à trois parties, structurée autour d'un médiateur et des médiés qui s'opposent, mais aussi des processus de restauration de liens rompus, corrompus ou devenus délétères, etc. Il resterait, toutefois, à prouver l'intérêt, autre que philosophique ou épistémologique, d'un tel effort. Ainsi que l'utilité de l'application des résultats.

II. La seconde serait de suivre un chemin aux antipodes du normatif. Elle consisterait à parcourir des corpora textuels pertinents, dans l'objectif d'expliciter certaines variations du terme. Cette attitude, volontairement descriptive, se révélerait, peut-être, plus modeste sur le plan théorique ; mais elle se revaloriserait rétrospectivement par l'amélioration de l'efficacité disciplinaire du terme de médiation. Elle serait probablement plus utile, peut-être plus efficace, au moins plus cohérente dans une optique terminologique traditionnelle. Sa condition de possibilité résulterait, ainsi, des techniques de traitement de corpora textuels et de la mise en valeur des résultats à travers une plateforme informatique convenable, ouverte, partageable à caractère contributif.

Un travail préliminaire de ce projet, travail tant bibliographique que de terrain, a toutefois montré que la construction d'un concept intégré et partagé de médiation conduirait à l'utilisation de caractères abstraits et plutôt spéculatifs, au point d'invalider d'éventuels bénéfices attendus sur le terrain d'un acte de médiation. Cette conclusion a été corroborée par la synthèse des travaux présentés lors de la conférence multidisciplinaire L'essentiel de la médiation. Vers un consensus sur le concept en sciences humaines et sociales de 2018 (DE GIOIA \& MARCON, 2020). Même en se limitant au domaine juridique, on observe que l'autonomie du concept de médiation est constamment remise en question par les besoins tant théoriques que méthodologiques et par l'apparition d'intérêts opportunistes. La vivacité éditoriale en témoigne : bon nombre de manuels apportent des définitions ainsi que des conceptualisations praxéologiques et procédurales fort diverses (par exemple, dans le contexte francophone, LASCOUX, 2001, SMETS-GARY \& BECKER, 2012, etc. ; dans le contexte italophone, BRUNI \& SitZIA, 2011, RUSCETTA et al., 2011, etc.).

Le projet débouche naturellement sur l'option d'une formalisation et d'une réalisation informatiques susceptibles de structurer les variations conceptuelles du terme "médiation", avec une claire référence à une discipline ou à une activité particulières. Il s'agirait donc de plusieurs onto-terminologies, chacune faisant la part des secteurs d'application du concept, mais au sein de la même logique 
informatique en matière d'implémentation. Respectueuses des domaines respectifs, ces onto-terminologies et le système informatique qui les soutient, viseraient à poser la pierre angulaire d'une réelle utilité, à la fois pratique et méthodologique : initier un abord documenté, coopératif et multilingue de la terminologie de la médiation.

Fondé sur un argument d'intelligibilité et d'efficacité, le projet a cherché à articuler, dans un premier temps, une approche en langue française. Mais ceci n'est pas limitatif.

\section{Méthodologie}

\subsection{Domaines d'application}

Le projet doit être entendu comme une «preuve de concept» («proof of concept »), au sens de "démonstration de faisabilité ». On rappelle qu'il s'agit d'une réalisation expérimentale, concrète mais volontairement circonscrite, dans l'objectif de mettre en évidence :

i. l'intérêt d'une idée, d'une démarche, d'une méthode, d'une technique, ou d'un savoir-faire et

ii. sa faisabilité.

Faisant le choix entre intensif et extensif, nous nous sommes limités, dans un premier temps, à cinq domaines dans lesquels le concept de médiation semble abondamment utilisé : les domaines

1. du droit,

2. de la formation,

3. de la communication,

4. du langage et

5. de la culture.

Ces cinq domaines sont accompagnés d'un sixième, dénommé sobrement « Médiation », sorte de domaine non pas forcément stéréotypé, mais, en quelque sorte, « de référence ». En effet, le projet a souhaité faire une petite place à un socle terminologique, certes pas « essentiel», mais au moins «typique ». Non pas pour s'essayer sur une sorte d' «ontologie terminologique de cœur», abstraite et de préoccupations plus ou moins philosophiques. La mise en place d'une « médiation a-disciplinaire de référence » pourrait encore répondre, nous semble-t-il, à une visée modeste et toujours défendable : dresser les contours de la typicité d'un ensemble de termes qui gravitent autour du concept de médiation (cf., comparativement, MEYER \& MACKINTOSH, 2000, Sinclair, 1991 et FELBER, 1987). On le sait, les «objets typiques» qui participent dans l'organisation de notre patrimoine sémantique ne sont ni les meilleurs, ni les plus fréquents, ni même les plus idéaux. Comme le montre excellemment KLEIBER (1991), ils sont simplement des objets sémantiquement dominants dans une culture, une pratique mais, surtout, dans un 
contexte linguistique. Ce sont des objets qui démontrent une représentativité optimale sous un principe d'économie cognitive.

Pour résumer, la visée du projet, descriptive, empirique et volontairement opératoire, s'énoncerait ainsi : concevoir une plate-forme susceptible d'intégrer les apports terminologiques venant de domaines divers, tout en réservant un socle de référence, provenant essentiellement (mais pas exclusivement) de l'étude première de De GioIA \& MARCON (2014). Mais, aussi, de BEN Mrad (2006).

Servant la cause interdisciplinaire, cette plate-forme, coopérative et ouverte (i.e. accessible sans frais d'entrée), possède une certaine pérennité dynamique, justement par l'amélioration additive qu'elle autorise et stimule, sous le mode de partage, désormais répandu et largement connu par le préfixe « wiki » : elle peut, en effet, accueillir des contributions terminologiques diverses, elle tolère les rectifications à tout niveau et reste extensible, pratiquement indéfiniment et de manière multilingue - comme on le verra dans la suite.

Une telle plate-forme se donne deux objectifs :

I. Constituer, tout d'abord, une sorte de «forum » pour des contributions terminologiques coopératives sur des termes qui composent le champ de la médiation. Offrir, ensuite, grâce à une ergonomie simplifiée permettant son appropriation aisée, un mode de travail qui capitalise sur la coopération d'une communauté de terminologues. Ce faisant, la plate-forme s'ouvre tant à l'agrégation de contenus qu'à leur consultation et à leur synthèse.

II. Proposer, ensuite, un mode éducatif sur le concept même de médiation. Autrement dit, offrir une manière de transmission et de partage d'un patrimoine sémantique, constitutif du champ de la médiation par la mise à disposition de moyens modernes pour élaborer des cours de terminologie.

Le projet s'engage, ainsi, en faveur d'une sorte de " médiation (sur le terme) de médiation ». (C'est dans ce sens qu'il serait convenable de comprendre le terme de «méta-médiation», du titre de l'article.) La matière sémantique de cette « médiation » est fondée sur le paradigme de la linguistique de corpus ; la matière fonctionnelle sur le paradigme informatique des systèmes d'auteur adaptatifs (cf., déjà, WÜSTER, 1981). En effet, d'un côté, divers corpora textuels sont convoqués pour appuyer une définition particulière d'un terme ; et, de l'autre, la plate-forme informatique autorise des ajustements et des accommodations à des profils d'utilisateurs, des demandes et des objectifs terminologiques variés.

\subsection{Termes retenus : territorialité sémantique du terme de médiation}

Les cinq (plus un) domaines retenus, ci-dessus, sont croisés avec une centaine de termes relevant du champ conceptuel large de la médiation. Elle est extraite de l'étude de DE GIOIA \& MARCON (2014). Le critère de sélection est généralement statistique, par domaine. On y rencontre des termes plutôt systématiques dans l'écologie sémantique de la médiation. Mais aussi d'autres, inattendus (comme " fantôme », « salle », " tableau », " réunion préliminaire », etc.) ou très techniques (comme MESORE, comédiateur, etc.). Qu'on nous pardonne cette licence : il ne 
s'agit que d'un choix pour la mise en épreuve du système développé. Dans tous les cas, cette liste n'est ni exclusive ni arrêtée : on peut non seulement l'amender (soustraire ou ajouter des termes), mais aussi y intégrer une terminologie relevant de langues autres que le français. De façon explicite, donc :

\begin{tabular}{|l|l|l|}
\hline accord & degré d'urgence & neutralité \\
\hline accord préalable & demande de médiation & norme juridique \\
\hline accueil & différend & option \\
\hline acteur & échange & position \\
\hline altérité & écoute active & première rencontre \\
\hline annulation & écouter & processus de médiation \\
\hline attente & émotion & protocole de médiation \\
\hline attention & empathie & question \\
\hline authenticité & entretien confidentiel & question clé \\
\hline besoin & entretien conjoint & question directe \\
\hline blocage & équidistance & question fermée \\
\hline cadre & équilibre & question ouverte \\
\hline centre de médiation & fantôme & récit \\
\hline changement & fausse question & reconnaissance \\
\hline clarification & humilité & reformulation \\
\hline climat & impartialité & reformulation miroir \\
\hline cohérence & indépendance & relation \\
\hline comédiateur & initiateur & représentation \\
\hline comédiation & initiative & résonance \\
\hline communication éthique & intérêt & responsabilisation \\
\hline communication non verbale & interrompre & responsabilité \\
\hline concession & jugement & réticence \\
\hline confiance & liberté politique & réunion préliminaire \\
\hline confidentialité & libre & salle \\
\hline conflit & liste des questions & séance \\
\hline conseil & maïeutique & secret professionnel \\
\hline conseil externe & mandat & situation juridique \\
\hline coopération & médiateur & sympathie \\
\hline courtoisie & tiódiation & tableau \\
\hline créativité & temps \\
\hline critère objectif & zone d'accord possible \\
\hline croyance & & \\
\hline définition & métive & tione \\
\hline
\end{tabular}

Tableau 1.2 : Liste des 98 termes retenus. 


\subsection{Définitions, ressources textuelles et relativité sémantique}

En croisant domaines (6) et termes (98), on obtient un tableau à deux entrées, avec quelque 600 cellules. Chaque cellule adresse un terme (en ligne) à un des 6 domaines (en colonne), comme sur la Figure 1.3-1.

Chaque cellule du tableau est un conteneur. En effet, il s'agit non pas d'un simple champ où on inscrit une information, mais d'un dossier qui peut être rempli de textes (sous format pdf), mais aussi, d'images, voire de fichiers audio et de vidéos, apportant des informations pertinentes sur le terme (indexé par la ligne) par rapport à un domaine (indexé par la colonne). La capacité de ce conteneur est presque illimitée : on peut y déposer autant de documents qu'on souhaite, sans restriction autre que celle du support informatique.

Ainsi, dans un premier temps, en termes de structure de dépôt, une telle matrice fonctionne comme un récupérateur ou, mieux, comme un intégrateur raisonné de documents, porteurs de " pépites terminologiques significatives ». Lors d'un travail critique (des articles, par exemple), ou d'une présentation (des cours, par exemple), on peut en extraire des documents ou des parties de documents, dans un objectif d'illustration, de documentation ou d'argumentation.

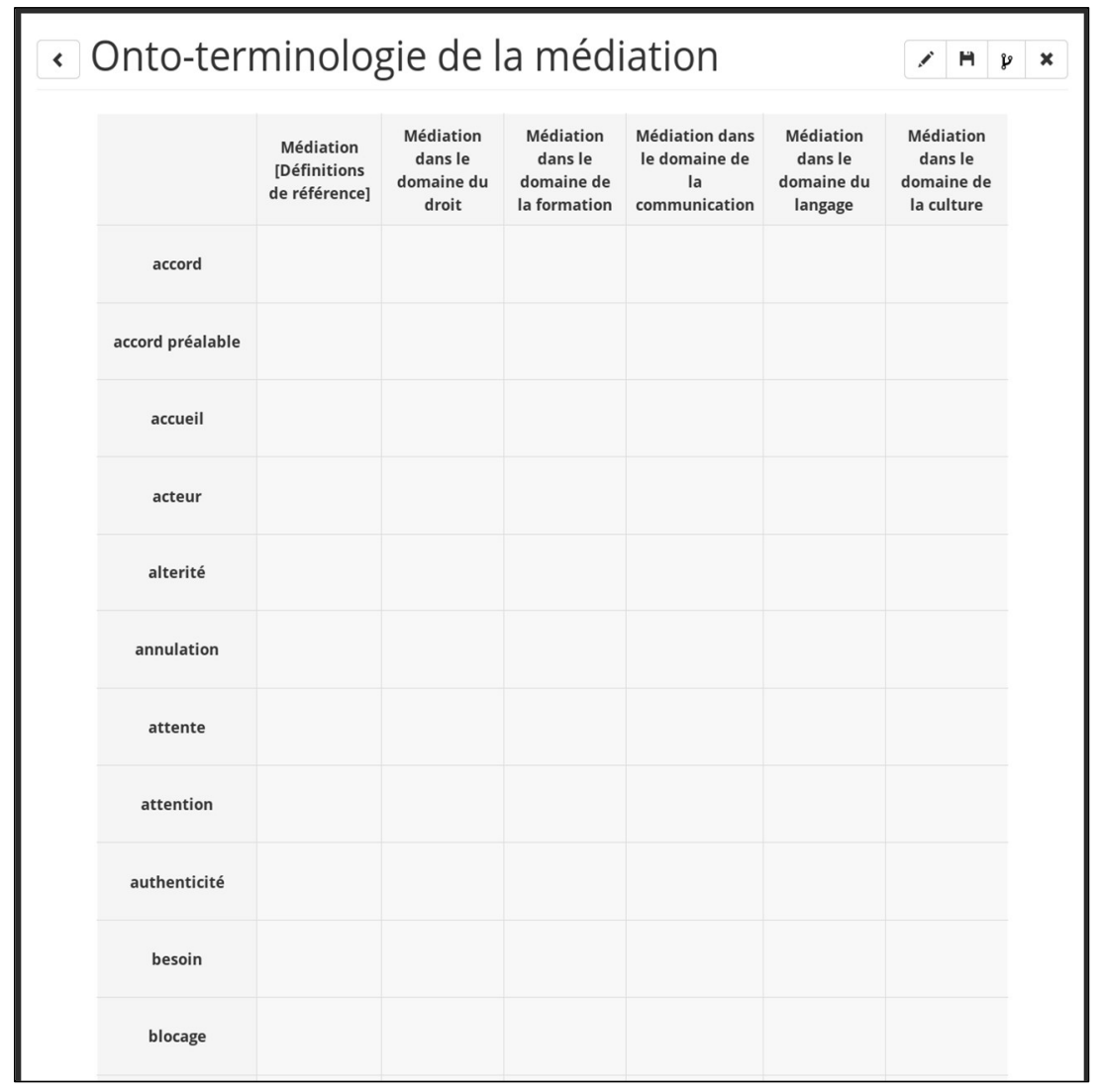

Figure 1.3-1 : Les croisements des domaines avec la liste des termes choisis. 
À des fins d'affinement, le projet s'est fixé comme objectif l'exploration terminologique de 7 termes, d'une manière plus approfondie :
i. conflit
ii. impartialité
iii. indépendance
iv. médiateur
v. médiation
vi. neutralité
vii. processus (de médiation)

Ainsi, dans la suite de cet article, on illustrera les travaux menés essentiellement à travers un tableau de dimension [7 x 6], comme le suivant :

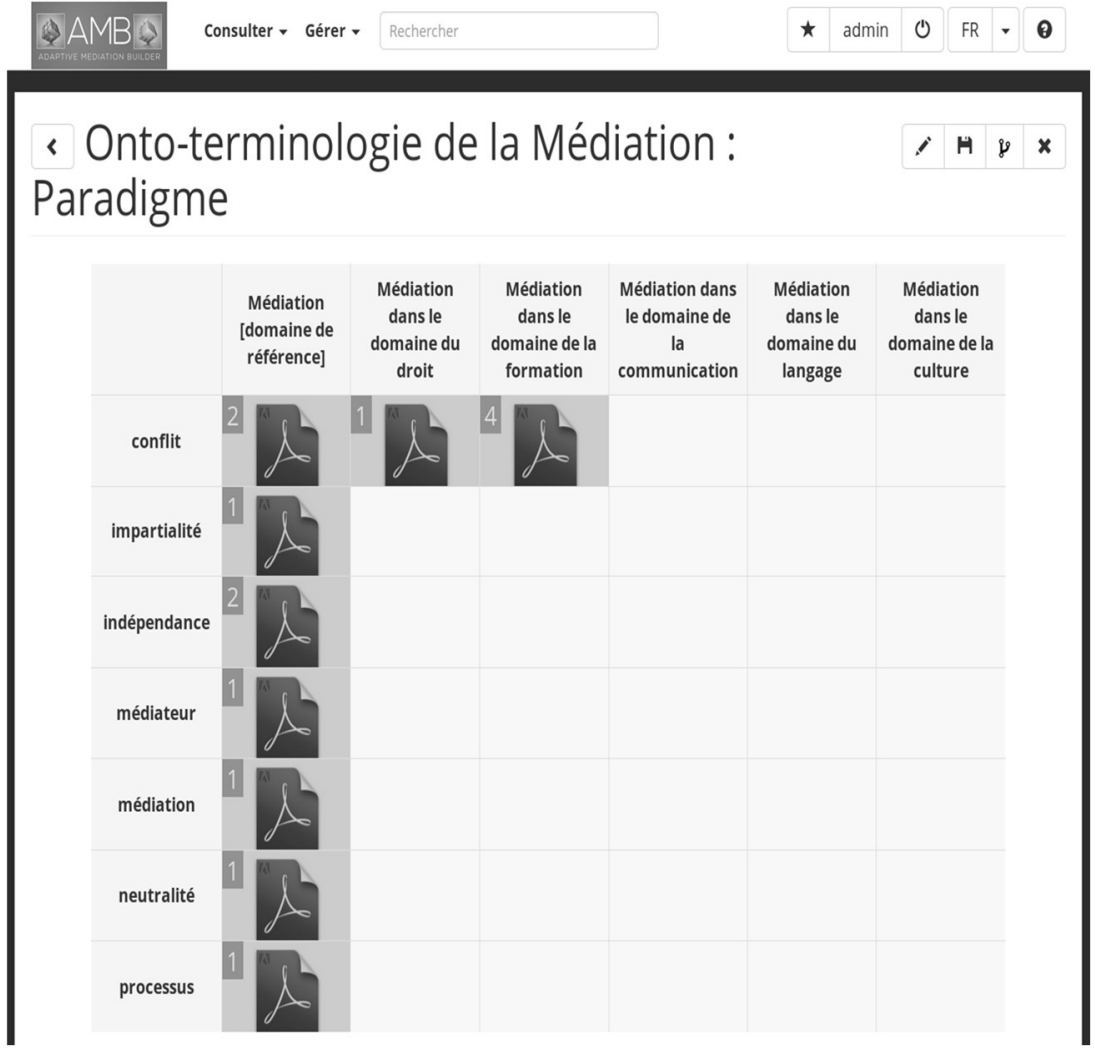

Figure 1.3-2: Matrice réduite du projet, limitée à 7 termes du domaine de la médiation (à gauche), avec l'ensemble des domaines choisis (en haut). Le nombre en haut à gauche de chaque cellule indique le nombre de ressources contenu à un moment donné de l'utilisation de la plate-forme (par exemple, 4 pour le terme « conflit » dans le domaine de la formation). 
Les textes sont généralement récupérés sur internet, au moyen d'une série de recherches multicritères, mettant en avant au moins le terme cible et le domaine visés.

Si le texte, après étude, se révèle porteur de clarifications sur le terme concerné, les parties qui s'y rapportent sont surlignées. Les textes déposés dans la matrice, comme celle de la figure précédente, offrent ainsi une image des enjeux terminologiques (cf. ci-dessous) :

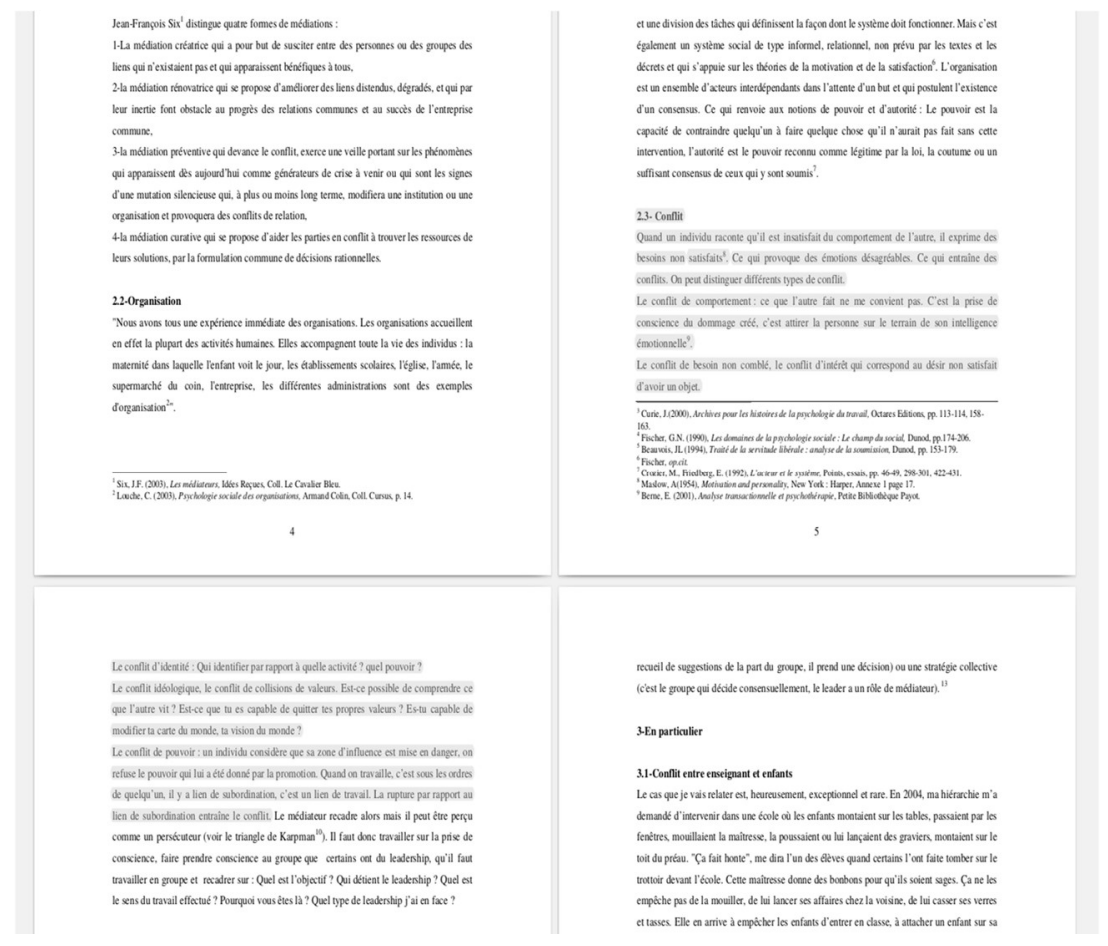

Figure 1.3-3 : Exemple d'un texte issu d'un quelconque corpus. Ici, il s'agit d'un texte de 22 pages («Conflit et médiation dans l'organisation scolaire », de Marc Lebraud, paru dans www.psychoressources.com, en 2008). L'utilisateur, jugeant intéressants (suivant ses propres critères) des éléments sémantiques concernant le terme « conflit », dans le domaine de l'éducation, les met en exergue.

Comme conteneurs, les cellules peuvent recevoir plusieurs documents se rapportant à un terme. Même s'il est possible de mettre toute forme de document, le projet s'est limité à des documents textuels.

Dans les cellules de la première colonne (appelée de " référence »), on trouve, en outre, une fiche terminologique (cf. la figure suivante) qui définit le terme étudié en référence à une conceptualisation a-domaniale (VÉZINA et al., 2009). Elle est aussi modifiable en forme, structure et contenu. 


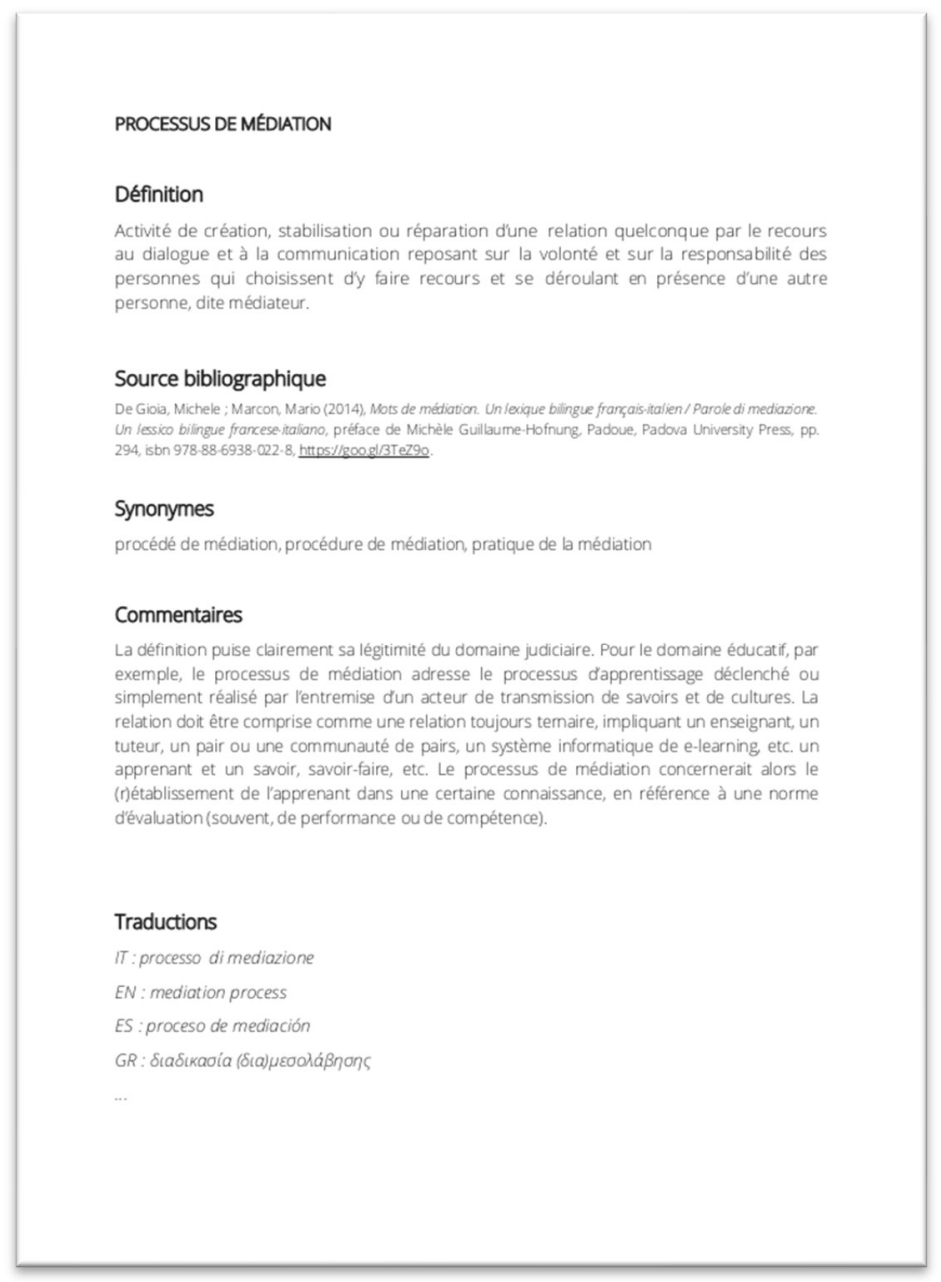

Figure 1.3-4 : Fiche terminologique de référence.

Il ne s'agit pas d'imposer une définition par domaine, mais, bien au contraire, de fournir un repère terminologique descriptif, reposant sur un corpus de textes pour chaque domaine. Clairement, plus le corpus évolue, plus la définition d'un terme s'affine.

\section{Terminologie, ontologie et onto-terminologie de la médiation}

Inscrit dans une logique en opposition par rapport aux démarches ontologiques, qu'on rencontre dans le domaine des représentations des connaissances en informatique, le projet d'une « onto-terminologie de la médiation » fait déplacer le souci ontologique vers la description du fait terminologique (cf. à ce propos, l'approche et l'analyse proposées par (KISTER et al., 2011) ainsi que la critique que les auteurs dressent sur les capacités d'une ontologie de rendre finement les relations sémantiques). Dans notre projet, il ne s'agit pas d'essentialiser le sens : une 
description terminologique s'impose, comme norme, le respect des pratiques et des intelligences collectives qui émaillent des pratiques. Il convient de rappeler que, en informatique, en représentation des connaissances plus spécifiquement, le terme «ontologie» désigne une modélisation et une représentation d'un domaine, informatiquement exploitable, dans lesquelles se reflètent les pratiques d'une communauté (autour d'un métier, par exemple). Cette représentation se fait au moyen de termes qui sont, dans le cadre informatique non pas des fragments de discours mais simplement des unités symboliques atomiques et calculables. Ces unités sont formellement reliées entre elles par de nombreuses relations binaires, auxquelles le concepteur de l'ontologie associe des propriétés variables, suivant la logique de description qu'il suit.

Selon la littérature consacrée, il s'agit d'une «explicit specification of a conceptualization » (GRUBER, 1993) ou encore d'un « engineering artifact, constituted by a specific vocabulary used to describe a certain reality, plus a set of explicit assumptions regarding the intended meaning of the vocabulary words 》 (GUARINO, 1998). Cette "réalité » peut être l'image d'un consensus d'une communauté ou le produit d'une proposition théorique. Une ontologie informatique est, au fond, une création formelle qui vise à définir un modèle capable : i. de décrire, ii. de reconnaître et iii. de prédire certaines entités, relations, processus, façons de faire ou comportements d'un univers donné, au moyen d'un vocabulaire clairement prédéfini et délimité, qui n'admet pas d'ambiguïtés ou de variations. À la base de telles constructions, on retrouve souvent des textes, mais aussi la perception et l'intuition de leurs créateurs (GUARINO et al., 2009). En d'autres termes, il s'agit d'une modélisation d'un ensemble de discours possibles sur une pratique.

À la différence d'une base de données, une ontologie possède généralement une finesse descriptive plus grande, dans la mesure où une ontologie est, au fond, la mise en place d'un ensemble de prédicats linguistiques, faisant un tout cohérent. Les relations binaires qui la constituent sont, effectivement, des formalisations logiques de prédicats ordinaires de la langue naturelle. Elles prennent la forme $a R b$, a et $\mathrm{b}$ étant des termes (qu'on appelle « concepts » en représentation des connaissances) mis en relation par un prédicat, représenté par la relation $\mathrm{R}$. Le nombre de telles relations peut être arbitraire. Au prix d'une réduction expressive, un tel univers devient logiquement calculable et démontre (contrairement aux bases de données standards) des capacités déductives.

Les ontologies sont des constituants importants dans le projet universel d'un web sémantique, et de la démarche des données ouvertes et reliées (« open \& linked data »). Elles jouissent déjà d'une histoire et d'une importante activité en matière de développement et de standardisation dans le cadre du W3C (World Wide Web Consortium: www.w3c.org). Divers langages de description (typiquement, le langage $o w l$ ), des standards de description et de représentation (typiquement, $R D F$ ), de logiques (typiquement, la f-logique de description) et de logiciels interactifs de conception d'ontologie (comme le logiciel libre Protégé), attestent d'une grande effervescence sur cette question à travers le monde.

Une ontologie a, donc, surtout, une certaine capacité inférentielle, qui résulte des propriétés formelles des relations qu'elle incorpore (transitives ou non, 
symétriques ou non, globales ou à exceptions, etc.). Ces propriétés se composent localement, assurent ou bloquent l'héritage des traits associés. L'intérêt d'une ontologie est de constituer un socle de connaissances structurées pouvant fonder divers services, suivant une façon particulière de comprendre et de réaliser une pratique ou un savoir-faire dans un domaine particulier (par exemple, le tourisme, la gastronomie, la muséologie, etc. ; et pourquoi pas, la terminologie). Ce faisant, elle tente de modéliser un mode opératoire d'un ensemble d'acteurs du domaine acteurs qui doivent y reconnaître leur activité. Elle explicite, en quelque sorte, une norme qui régit un savoir-faire, sous un mode logique. C'est-à-dire, calculable.

Il n'y a donc pas de «bonne » et de «mauvaise » ontologie a priori. Il y a seulement « ontologie adéquate » ou " inadéquate », par rapport à une pratique et une communauté. Une ontologie peut être inadéquate pour un type de pratique pour une communauté (parce qu'elle ne travaille pas de cette manière), alors qu'elle peut l'être très bien pour un autre, pour des raisons opposées. La remarque est capitale pour notre argument en matière de terminologie de la « médiation».

De l'autre côté, une ontologie extensive (i.e. possédant un nombre élevé de termes et de relations), n'est pas nécessairement meilleure qu'une autre, plus restreinte. L'intérêt d'une ontologie réside, exclusivement, en sa capacité de démontrer un comportement plus ou moins en conformité avec une façon de comprendre une praxis particulière. L'ontologie cherche à établir le schéma procédural des concepts, des relations et des actions composant cette praxis, actions qui doivent pouvoir être représentées formellement et être mises en synergie cohérente et efficace, par une voie logique. Il ne s'agit pas d'une ontologie au sens philosophique du terme, visant l'être dans sa globalité ou son intégrité transcendantes. Mais d'un emprunt terminologique, généralisé et importé par les informaticiens, spécialistes en représentation des connaissances, pour représenter une réalité locale, pratique, événementielle, procédurale et normée. D'ailleurs, une ontologie extensive de plusieurs centaines de termes implique un coût élevé en matière de conception - ne serait-ce que pour établir et maintenir sa cohérence. Elle devient, de surcroît, fragile et difficile à faire évoluer, dans la mesure où elle exige d'établir, à toute modification, les critères de sa cohérence logique globale.

La différence entre ontologie et onto-terminologie, dans notre cas, entre une ontologie formelle de la médiation et une onto-terminologie des domaines de la médiation, doit être identifiée moins, peut-être, à la nature des entités examinées plutôt qu'aux attentes associées à sa réalisation. Certes, une onto-terminologie, qui se concentrerait surtout sur des termes, en espérant capter la charge sémantique portée par eux pour, éventuellement, modéliser une pratique (dans notre cas, d'une certaine pratique de la médiation dans un domaine spécifique), pourrait encore afficher quelques-unes des exigences formelles usuelles d'une ontologie informatique ordinaire. Cependant, une onto-terminologie ne vise pas l'augmentation de la base des connaissances par inférence : le sens, n'est pas une déduction ni une propriété immanente d'un terme. Et la normativité dont peut attester une pratique (mais aussi, la normativité, en général) ne saurait être captée au moyen d'un petit ensemble de prédicats, organisés logiquement.

Mais supposons que cette première contestation reste mineure. Nous avons déjà fait observer que, depuis les travaux du colloque sur «l'essentiel de la médiation » 
(DE GIOIA \& MARCON, 2020), la mise au clair du nomadisme extrême de la notion et de la grande versatilité des pratiques, rend une telle tâche de peu d'intérêt.

Quoi qu'il en soit, la réfutation la plus importante d'une ontologie classique de la médiation vient des objectifs poursuivis dans ce projet. En clair, le projet d'une onto-terminologie de la médiation vise à mettre sur pied un mode opératoire servant le dialogue entre domaines concernés par la médiation. Loin de vouloir figer les choses pour une prétendue efficacité, il s'agit bien de mettre en évidence les déviations, les écarts, les détours, les inflexions et autres « dissidences » du sens et de son utilisation, dans l'objectif de contribuer à la mise en place d'une conscience sémantique de tolérance et d'intercompréhension entre pratiques de médiation. L'onto-terminologie de médiation préconisée par notre projet sert donc la volonté de comprendre avant de s'attacher à une quelconque prétention d'agir.

" Médiation sur la médiation », ce projet cherche, enfin, à mettre en exergue des présentations comparatives et structurées de «tranches sémantiques », issues de la « vie » du concept de médiation dans des niches de pratiques diversifiées. L'objectif est de décrire aussi largement et finement que possible et de présenter clairement leurs altérations et leurs contrastes sémantiques. On illustrera mieux ces dernières affirmations dans la suite (section suivante), en commentant les possibilités de réaliser des présentations à des fins de transmission des connaissances et des cultures médiatives.

Pour résumer : il serait illusoire, pour notre projet, d'imaginer un consensus autour de la centaine de termes choisis, de la section 2.2. Non seulement à cause de la «jeunesse » du concept de médiation dans certains domaines, jeunesse qui lui confère, en quelque sorte, une « impétuosité sémantique ». Mais, surtout, parce qu'il n'y a pas de demande précise. Aucun acteur de médiation dans les cinq domaines que nous considérons n'exprime des besoins nécessitant la mise en place d'un système logico-déductif pour bâtir des services pouvant seconder son travail. Du moins, pas pour l'instant. Et on ne construit pas un système sur des demandes inexistantes ! Ainsi, le recentrage de l'onto-terminologie sur des préoccupations terminologiques traditionnelles semble plus viable et, peut-être, sur le plan du partage des connaissances, plus salubre.

En conséquence, l'outil informatique choisi ne vise pas des performances logiques, ni inférentielles en matière sémantique ; mais seulement l'institution de modes coopératifs, accessoirement critiques et comparatifs, au moyen d'un support informatique moderne, agrégeant des «actifs terminologiques». Ces «actifs» constituent la richesse terminologique de chaque domaine choisi, suivant les critères que se donne l'utilisateur du système. La communauté des pratiques sous-tendant un tel objectif est donc une communauté d'intérêts, ou, si l'on veut, une « communauté imaginaire », au sens de ANDERSON (1983) : celle de toute personne se reconnaissant dans l'intérêt porté pour un abord critique du champ sémantique de la médiation dans quelque domaine.

C'est en ces termes que l'on doit comprendre la visée « onto-terminologique » du projet. 


\section{Volet informatique : conception, modélisation et implémentation}

Telle que préconisée dans les sections précédentes, l'implémentation informatique de l'onto-terminologie de la médiation s'est fondée sur le système AMB (« Adaptive Mediation Builder» : www.edu3d.enstb.org ; cf. ANTIN et al., 2013 et 2016, ANTIN \& KANELLOS, 2015, KANELLOS, 2019). AMB a été choisi dans la mesure où il présentait des fonctionnalités pouvant satisfaire les exigences déjà évoquées. Il s'agit d'un système conçu et réalisé au sein du département Informatique de l'IMT Atlantique. En peu de mots, AMB est un système de médiation polyvalent, pouvant se montrer i. tolérant face à des visions concurrentes concernant un sujet et ii. adaptable à diverses spécificités de la réception. Dans notre cas, la diversité que peut prendre une telle réception renvoie aux différents sens des termes, envisagés, à chaque fois, dans des domaines variés d'application de la médiation.

\subsection{Le système AMB : son intérêt pour l'onto-terminologie de la médiation}

AMB est un outil de travail conçu pour assister à la conception de certaines formes de médiation. Plus spécifiquement, pour aider à la construction d'un « discours » commun, sur un thème ou une question, tout en respectant le niveau et les attentes de ceux à qui on s'adresse. Ce « discours » prend souvent la forme d'une co-construction, par la participation éventuelle de divers acteurs. Grâce à la médiation que le système permet, ces acteurs cheminent ensemble, au sein d'un schéma de synergie commun en s'appuyant sur des catégories de pensée et d'action qu'ils se définissent au moyen du système.

Mais, par-dessus tout, et plus généralement, AMB met à disposition des moyens techniques pour développer des projets d'accompagnement et d'assistance en lecture, en interprétation et en compréhension (d'un thème, d'un terme, d'un concept, d'une situation, d'un objet, etc., relevant de la médiation). Telle est, en effet, l'intention du projet que nous décrivons ici : l'accompagnement, le soutien et la volonté de désambiguïsation des termes satellisés par le concept de médiation. On le sait, un des vecteurs majeurs susceptible de palier les problèmes posés par l'ambiguïté, c'est précisément la paraphrase i.e., la mise au point et la prise en compte d'un discours explicatif, alternatif ou complémentaire. AMB permet, en effet, une action sémantique additive, offrant lieu et exposition à des voies sémantiques, qui approfondissent ou qui s'opposent à celles déjà en place, et ceci, tout en respectant les spécificités d'emploi des termes dans des domaines différents. Par ailleurs, en matière de terminologie, $\mathrm{AMB}$ assure et stimule des médiations à des niveaux divers d'implication de la part des participants, qu'ils soient dans la position des médiés ou des médiateurs. Sa conception se fonde sur la notion de "grain » : un grain est une ressource d'information ayant une certaine autonomie sémantique. Dans le cadre de notre projet, ces ressources sont des textes incluant des indications terminologiques concernant un terme ou, éventuellement, des fiches précisant la définition d'un terme. Ces grains peuvent être combinés à volonté pour construire des listes, qui retracent des univers sémantiques propres à un domaine. Ces listes peuvent prendre la forme d'une sériation de textes qui viennent appuyer une définition terminologique (intertextualité). Elles s'organisent suivant : 
i. des points de vue complémentaires; ce qui correspond, dans notre cas, aux tranches sémantiques apportées au concept de médiation par les termes que ce dernier convoque dans un domaine particulier (98, dans une vision extensive de ce projet; ou 7, dans une volonté d'exemplification plus poussée) ;

ii. des niveaux d'approfondissement ou des domaines d'appréciation multiples : l'essentiel, l'accessoire, l'extensif, etc. ; mais aussi des manières de lire, des aspects, des topiques, etc. ; ce qui correspond, dans notre cas, aux domaines retenus pour la démonstration de la faisabilité du projet ( $6 \mathrm{au}$ total) ;

iii. des variantes de présentation éclectiques (réécritures, séquences alternatives, mise en avant des incidences du genre ou du style, etc.) ; ce qui correspond, dans notre cas, à la variation eidétique des textes enregistrés dans chaque cellule (le nombre étant illimité).

AMB est destiné tant à des médiateurs, dont l'objectif est de créer des contenus, qu'à des récepteurs divers, dont le but pourrait être de s'informer, de découvrir, d'étudier, d'explorer, etc. AMB peut, ainsi, venir en support de plusieurs objectifs d'utilisation (scientifiques, éducatifs, culturels, etc.), en phase de préparation, de réalisation ou de capitalisation des contenus sémantiques qu'il accueille.

\subsection{Description succincte du système AMB}

\subsubsection{Usages génériques : consultation et gestion}

Le système $\mathrm{AMB}$ a été conçu pour être adaptable tant au niveau du contenu qu'à celui de sa présentation. Les fonctionnalités du système sont partagées en deux usages principaux : la consultation et la gestion. Dans notre cas, il s'agit de consulter des ressources terminologiques et de les gérer, essentiellement dans l'objectif de les présenter de façon critique.

\begin{tabular}{|l|l|l|l|l|l|l|l|}
\hline AMB & Consulter - Gérer - Rechercher & $\star$ & admin & $\mathcal{O}$ & $F R$ & $\bullet$ \\
\hline
\end{tabular}

Figure 3.2.1-1 Les fonctionnalités génériques du système AMB : Consulter et Gérer. La connexion est ici faite en français en tant qu'administrateur.

- La rubrique «Consulter» donne accès à toutes les ressources qui se trouvent dans la base de données du système. À l'heure où nous rédigeons ces lignes (juin 2019), le corpus de ces textes contient quelque 300 ressources.

- La rubrique « Gérer » permet d'accéder à une suite de modules qui, d'un côté, aident le concepteur à mettre en forme un cadre général pour ses scénarios de médiation et, de l'autre, lui permettent de dresser des présentations, d'affiner la définition du contenu terminologique ou de modifier la structure des éléments de la présentation. 


\subsubsection{Aperçu de quelques fonctionnalités de base}

AMB offre une palette étendue de fonctionnalités qu'il serait impossible de décrire dans cet article, dont l'objectif n'est que de défendre un argument d'utilité et de faisabilité ainsi que d'en illustrer quelques éléments marquants. Nous allons nous limiter à quelques-unes seulement, celles, précisément, qui nous paraissent offrir une image plus claire de la valeur ajoutée du système dans nos préoccupations terminologiques.

L'utilisateur a la possibilité non seulement de parcourir la base des ressources en tant que simple lecteur, mais aussi de l'augmenter, en ajoutant des ressources complémentaires.

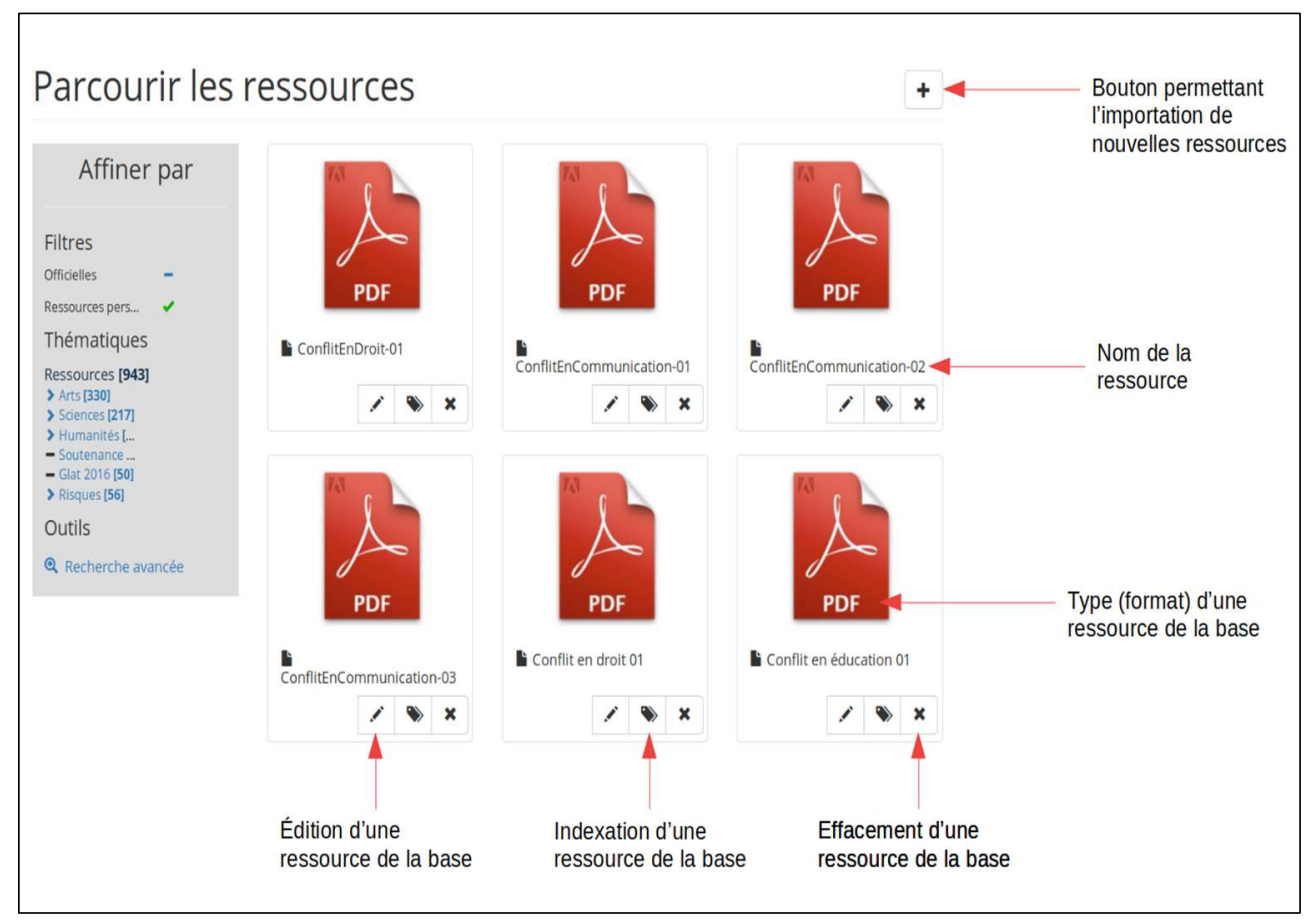

Figure 3.2.2-1 : Consultation du contenu enregistré dans l'espace personnel. Chaque ressource textuelle est accompagnée par un menu qui autorise l'utilisateur à avoir accès à l'édition de ses métadonnées (titre, description, motsclés, type, taille, date de création et de modification, etc.), à sa suppression et à son indexation. Le bouton « Importer » (symbolisé par un «+ " en haut, à droite) permet d'importer une ressource et de la rajouter à sa propre collection.

L'indexation des ressources est réservée à un utilisateur ayant des droits de gestion augmentés (permission spécifique d'intervention dans la gestion du système). L'indexation individuelle des ressources se réalise à partir de la rubrique « Gérer ». Dans AMB, elle suit une logique taxinomique, en contraste, bien entendu, avec la conception usuelle des ontologies actuelles, qui sont généralement des graphes. Dans notre cas, on adopte une organisation arborescente qui correspond à l'organisation de la base de données des ressources. Cette organisation se doit d'être cohérente avec la philosophie du framework de développement (Symfony). 
Toutefois, AMB permet la prise en compte de visions taxinomiques diverses, même incompatibles entre elles, qui reflètent les représentations des domaines et des usages proposées par des utilisateurs différents.

\subsubsection{Les ressources, les topiques, les matrices et les présentations : les quatre stades principaux de la mise en valeur sémantique d'un terme.}

La conception du système AMB se fonde sur une logique d'assistance et d'accompagnement d'une action interprétative portant sur un terme. Dans notre cas, il s'agit d'interprétations visant la terminologie de la médiation (mettre au clair les interprétations différenciées d'une écologie de termes dans divers domaines d'utilisation de la médiation).

Pour servir cette logique, le système repose sur une séquence de quatre actions. La figure suivante illustre le protocole typique d'utilisation du système AMB de l'étude d'un terme jusqu'à sa présentation raisonnée :

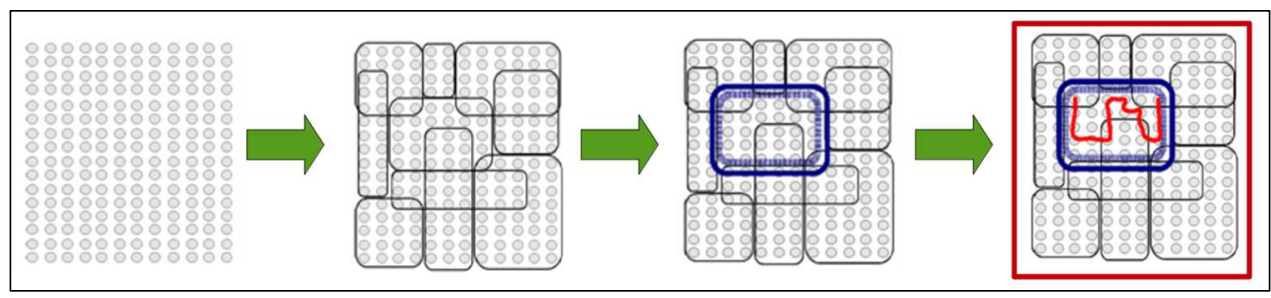

Figure 3.2.3-1: Stades d'utilisation de AMB. Par ordre de succession: i) constitution de la base des grains (i.e. des textes), bruts, ii) classification des grainstextes dans des topiques adéquates (ici, une seule topique, celle de la médiation), iii) construction d'une matrice dans l'objectif d'enrichir, avec des métadonnées, un certain nombre de ces grains-textes et iv) mise en place d'une présentation des résultats sous forme d'une liste des textes qui appuient un argument terminologique.

Les deux premières opérations, fondamentales, sont de constituer la base des ressources textuelles et de les classer dans une topique. À travers un dispositif interactif simple, l'utilisateur télécharge (upload) dans le système, un par un, les textes qui lui paraissent (suivant ses propres critères) pertinents. Tout critère est a priori légitime. À chaque ressource, on donne un titre (nom du fichier, tel qu'il apparaîtra dans AMB), et, éventuellement, un descriptif textuel ainsi qu'une série de mots-clés (cf. la figure suivante).

Enfin, on classe la ressource ainsi indexée dans la topique relative. Pour les besoins du projet, une topique "Médiation » a été créée de manière ad hoc lors d'une étape précédente. 
Description

Mots-clés

Thématique

\section{-Écologie \\ - - lle de Sein}

--Becyclage

-Physique

-Mathématiques

-Économie

-Informatiqu

Humanités

-Médiation

Figure 3.3.2-2 : Téléchargement des ressources dans la base du système et classification dans la topique « Médiation ».

On arrive, ensuite, à la troisième opération, sans doute la plus importante : la délimitation d'un ensemble cohérent de ressources-textes, pouvant présenter une valeur argumentative. En effet, une fois la base des ressources constituée, il s'agit d'en choisir un certain nombre, jugées pertinentes pour un objectif d'exploration terminologique, et de les enrichir avec des métadonnées. Ces métadonnées concernent :

i. la tranche sémantique concernée, i.e. le point de vue instancié par un terme particulier du champ sémantique de la médiation (un parmi les 98) et

ii. le niveau d'approfondissement sémantique souhaité, qui n'est que le domaine dans lequel le terme est envisagé (parmi «droit», «formation», " communication », « langage » et « culture »). Au fond, il est ici question d'une spécification classificatoire complémentaire.

Le cœur du système est précisément un générateur de matrices de telles métadonnées. Toute matrice représente le cadre conceptuel sur lequel s'appuiera dans la suite toute mise en évidence d'une interprétation concernant le sens d'un terme. Elle fixe une méthodologie de lecture capable d'assister l'utilisateur dans son parcours d'interprétation et de compréhension d'un terme, au sein de son domaine d'application, voire dans un objectif comparatif.

Une matrice n'est donc pas un canon; elle reflète seulement une vision particulière sur un corpus, celle, précisément, de son (ou de ses) créateur(s). Elle modélise, dirions-nous, le premier acte de toute étude : la collecte du corpus et de son organisation. Elle correspond à un espace de travail comprenant un ensemble de textes, qui délimite les frontières d'une lecture, d'un discours, d'un argument... 
Structure ontologique légère, elle cherche à modéliser des éléments interprétatifs de fond de son (ou de ses) créateur(s), pour des objectifs de clarification sémantiques.

Une matrice est flexible et accepte des reconfigurations multiples : on peut y définir autant de points de vue et de niveaux que l'on veut, renommer, changer et éditer des parties, etc. Par ailleurs, le système accepte un nombre arbitraire de matrices. Dans la version paradigmatique et opératoire de notre projet, la matrice est de dimension $[7 \times 6]$ comme sur la figure suivante. On peut éditer à loisir les champs de manière simple et intuitive. Il ne s'agit pas d'opérationnaliser la pertinence scientifique de l'utilisateur, mais seulement d'en offrir des moyens pour contribuer à un enrichissement des ressources en vue d'un usage coopératif.

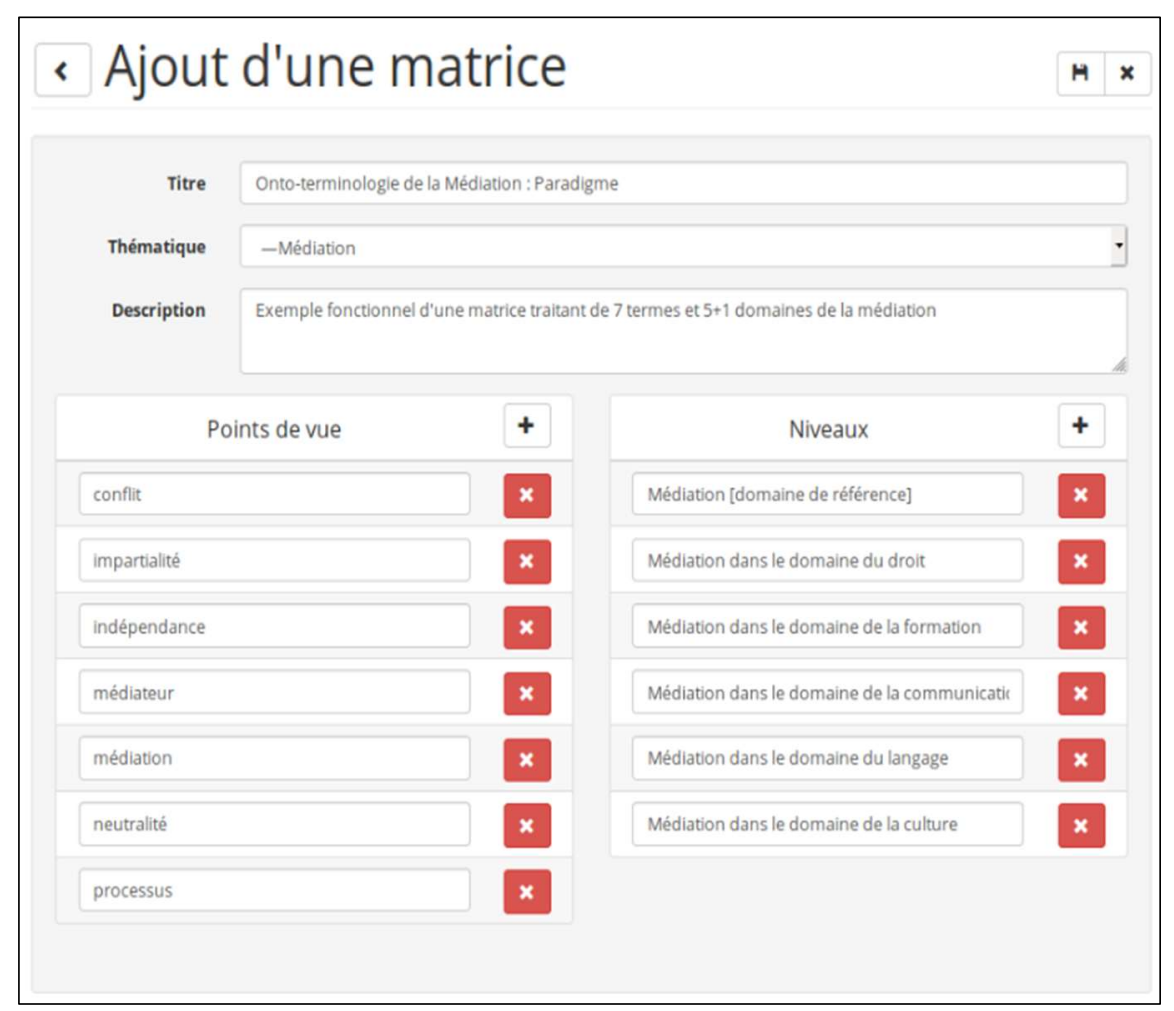

Figure 3.2.3-3 : Interface de création de matrices.

L'étape suivante concerne le « remplissage » de la matrice avec des ressources jugées pertinentes par l'utilisateur, afin de soutenir sa propre vision terminologique. Ici encore, les critères sont ceux de l'utilisateur. Le système offre un moyen pour structurer l'intertextualité sous-tendant un terme dans un domaine choisi, mais ne norme pas ces critères. La figure suivante illustre un moment de ce remplissage. À partir de la liste des ressources déposées dans la topique « Médiation » (en bas, à droite), on opère des glisser-déposer successifs, en consignant, dans chaque cellule [terme $\times$ domaine] une ou plusieurs ressources textuelles. Chaque cellule de la matrice est un conteneur qui peut comporter un nombre arbitraire de ressources. Par ailleurs, une même ressource peut appartenir à plusieurs cellules. 


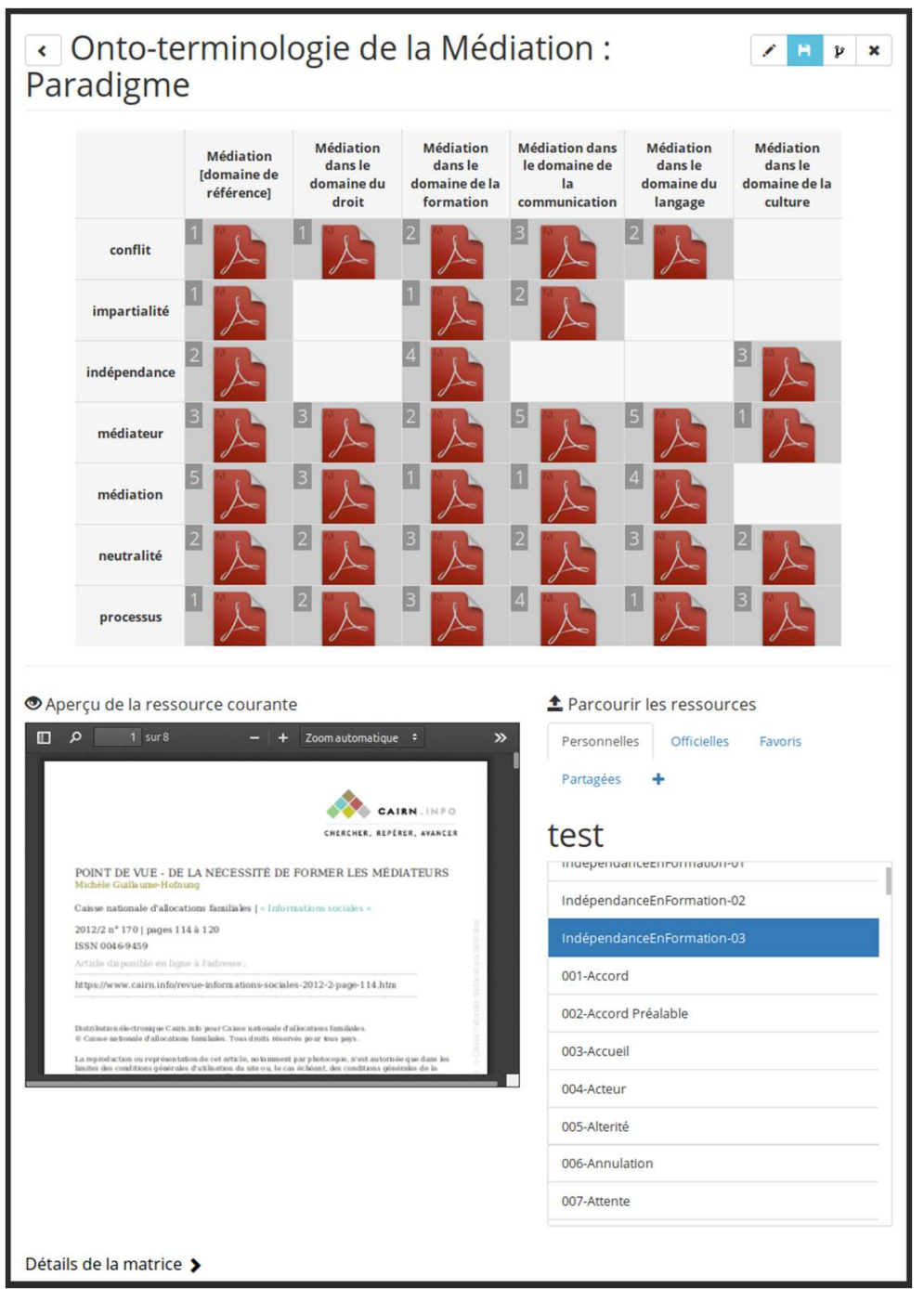

Figure 3.2.3-4 : Remplissage d'une matrice. En haut, la matrice en voie de remplissage avec des textes, issus de la base des ressources. Certaines cellules sont encore vides, d'autres contiennent plusieurs ressources (nombre en haut à gauche dans chaque cellule). En bas à droite, la liste des ressources. En bas à gauche, un lecteur de ressources, qui offre, le cas échéant, un aperçu de la ressource avant qu'elle soit déposée dans une des cellules de la matrice (ici, on visualise la ressource « IndépendanceEnFormation-03»).

En s'appuyant sur une matrice, on peut enfin produire des présentations (quatrième étape dans le processus ; cf. Figure 3.2.3-1). Une présentation consacre le dernier stade de l'utilisation de AMB : la mise en évidence et l'exposition raisonnée d'un sens. Sur la figure suivante, on illustre le processus de création d'une présentation; elle concerne, en l'occurrence, les différences sémantiques du terme «médiateur » dans les domaines de la formation, du langage et de la culture. Ces 
différences ne sont pas toujours évidentes, bien entendu. Et leur appréciation n'est pas la même pour tout utilisateur. Le système offre seulement la possibilité de comparer divers corpora (intra- ou inter-domaniaux). Mais il ne remplace pas le travail terminologique.

Toute présentation peut toujours recevoir des compléments pour un travail terminologique d'approfondissement. En effet, on peut toujours ajuster la présentation au niveau des récepteurs voire lui assigner des exigences pédagogiques: est-elle de base et nécessaire ou bien optionnelle et complémentaire ? Concrètement, en choisissant une ressource, on peut décider si elle appartient à la première catégorie (présentation initiale, de base) ou à la seconde (présentation suggérée, complémentaire), par simple clic. Sur la figure suivante, les codes « $\sqrt{ }$ vert » et « $\sqrt{ }$ orange » correspondent, respectivement, à la liste initiale, soit la liste de ressources nécessaires, et à la liste suggérée, soit la liste de ressources complémentaires. On peut, bien entendu, à tout moment, modifier les choix, en particulier, faire basculer une ressource depuis une liste vers l'autre, voire la supprimer. 


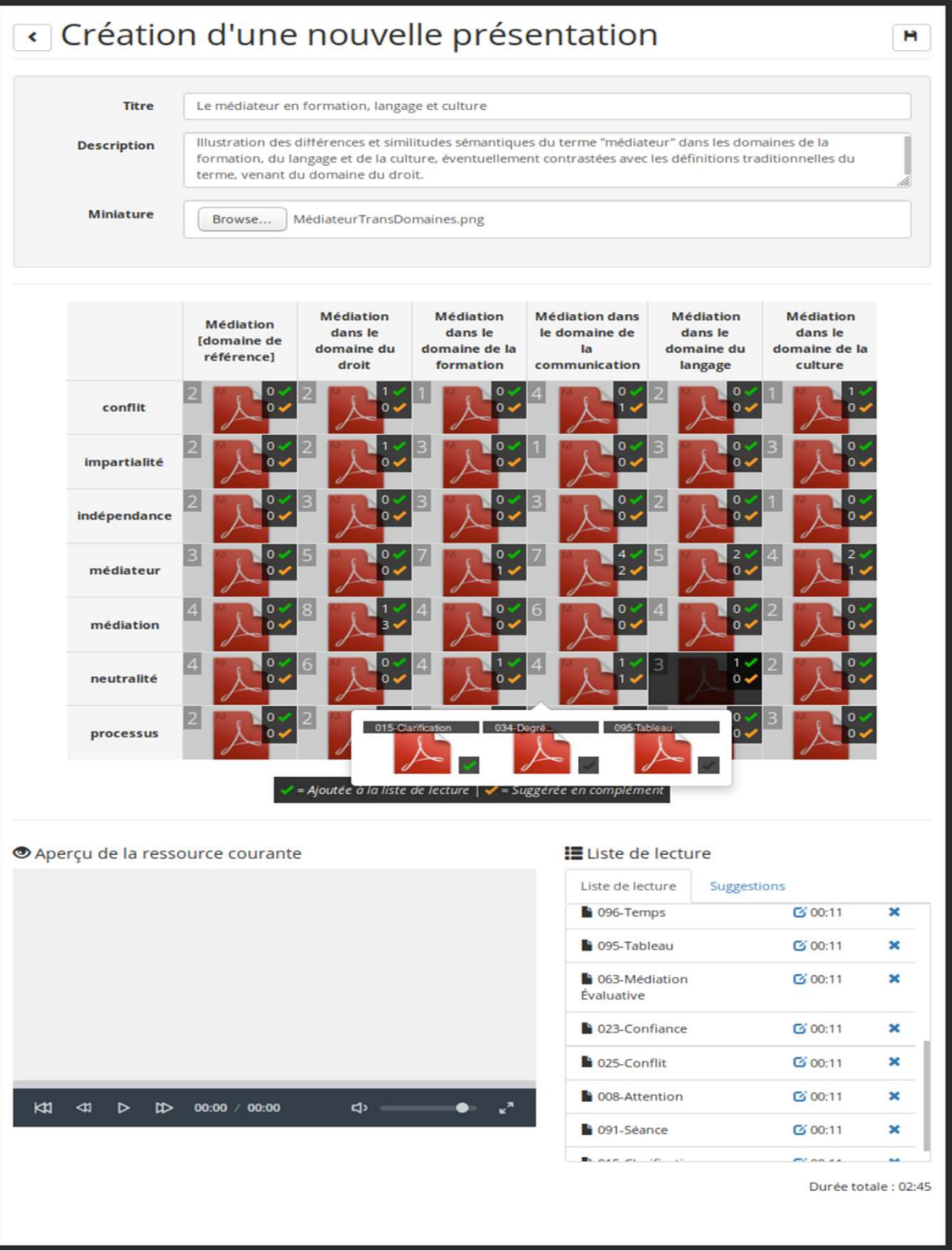

Figure 3.3.1-5 : Création d'une présentation. Ici, on s'intéresse, par exemple, au terme « médiateur » dans les domaines de la formation, du langage et de la culture. On peut modifier l'ordre des ressources dans la présentation, ainsi que le crédit temps alloué à leur exposition (par défaut, à 11 secondes), leur statut en matière d'importance dans le discours présenté (nécessaire ou optionnelle), intervertir, supprimer, rajouter, dédoubler, etc. On illustre le moment où l'on a cliqué sur le terme «impartialité » dans le domaine « Langage », pour choisir un grain textuel, jugé pertinent, (parmi les trois contenus dans la cellule) afin d'illustrer le sens du terme «médiateur». On peut à tout moment visualiser une ressource grâce au lecteur (en bas à gauche). Enfin, il est possible d'associer une image miniature à une présentation (cf. figure suivante). 
Une fois créée, la présentation trouve sa place dans la base des présentations du système.

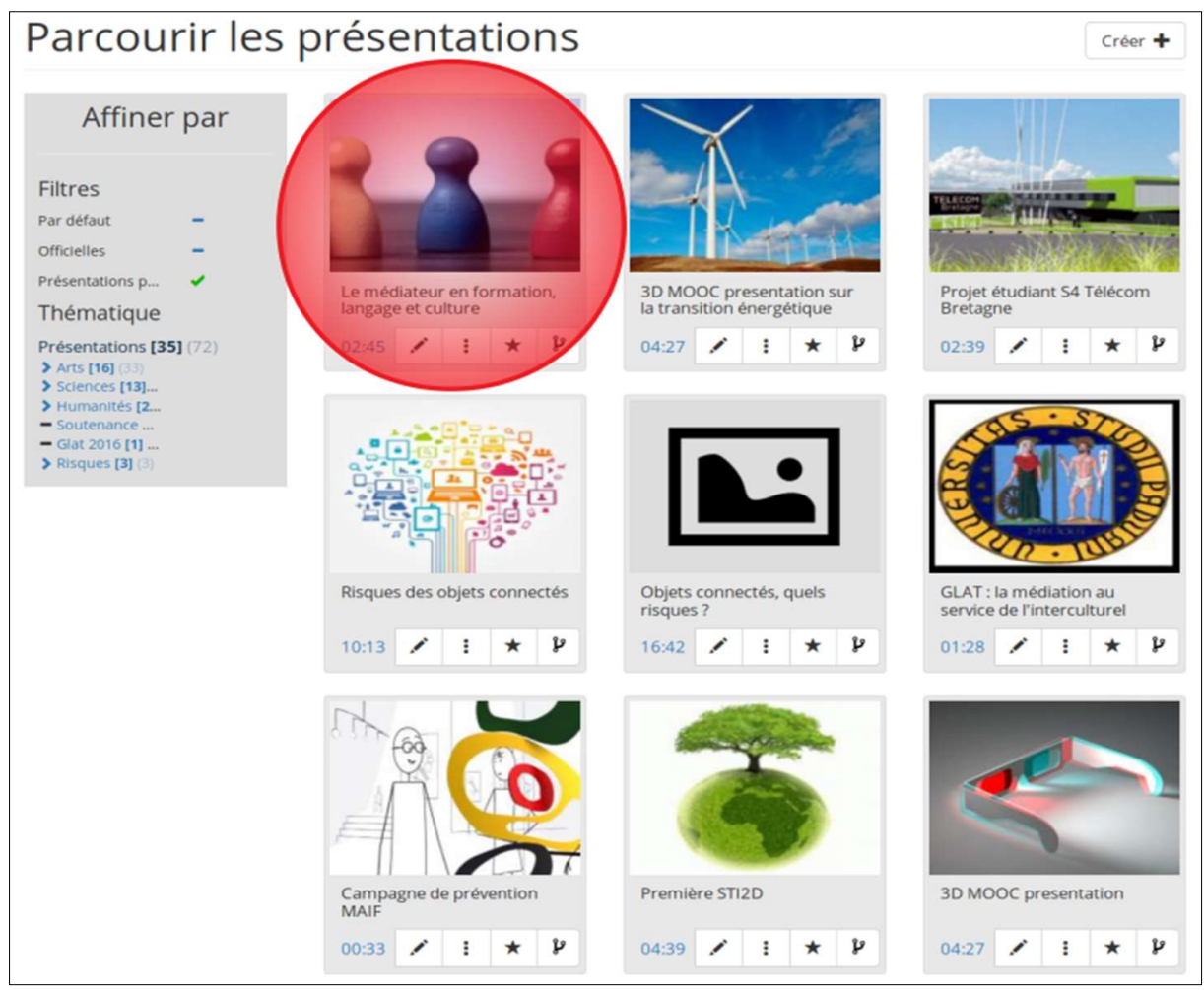

Figure 3.3.1-6: La présentation créée à partir d'une comparaison trans-domaniale du sens du terme « médiateur ». Elle est identifiée par son titre et, le cas échéant, l'image miniature associée.

Désormais, elle peut être utilisée pour soutenir une étude, un essai, une exploration, une réflexion, etc., envoyée à d'autres pour consultation, former la base pour un cours, etc. Plus généralement, elle peut être intégrée au sein d'une action de transmission et de partage de savoirs particulière, en présentiel ou à distance, de façon synchrone ou asynchrone, voire être partagée, copiée et transformée, de façon plus ou moins importante, pour servir des objectifs apparentés.

En mode de réception, la présentation prend la forme d'une liste de ressources (textes, dans notre cas) qui peuvent se dérouler soit automatiquement, suivant un ordre et une durée choisis, soit par intermittence. En matière de visualisation, elle prend, concrètement, la forme suivante : 


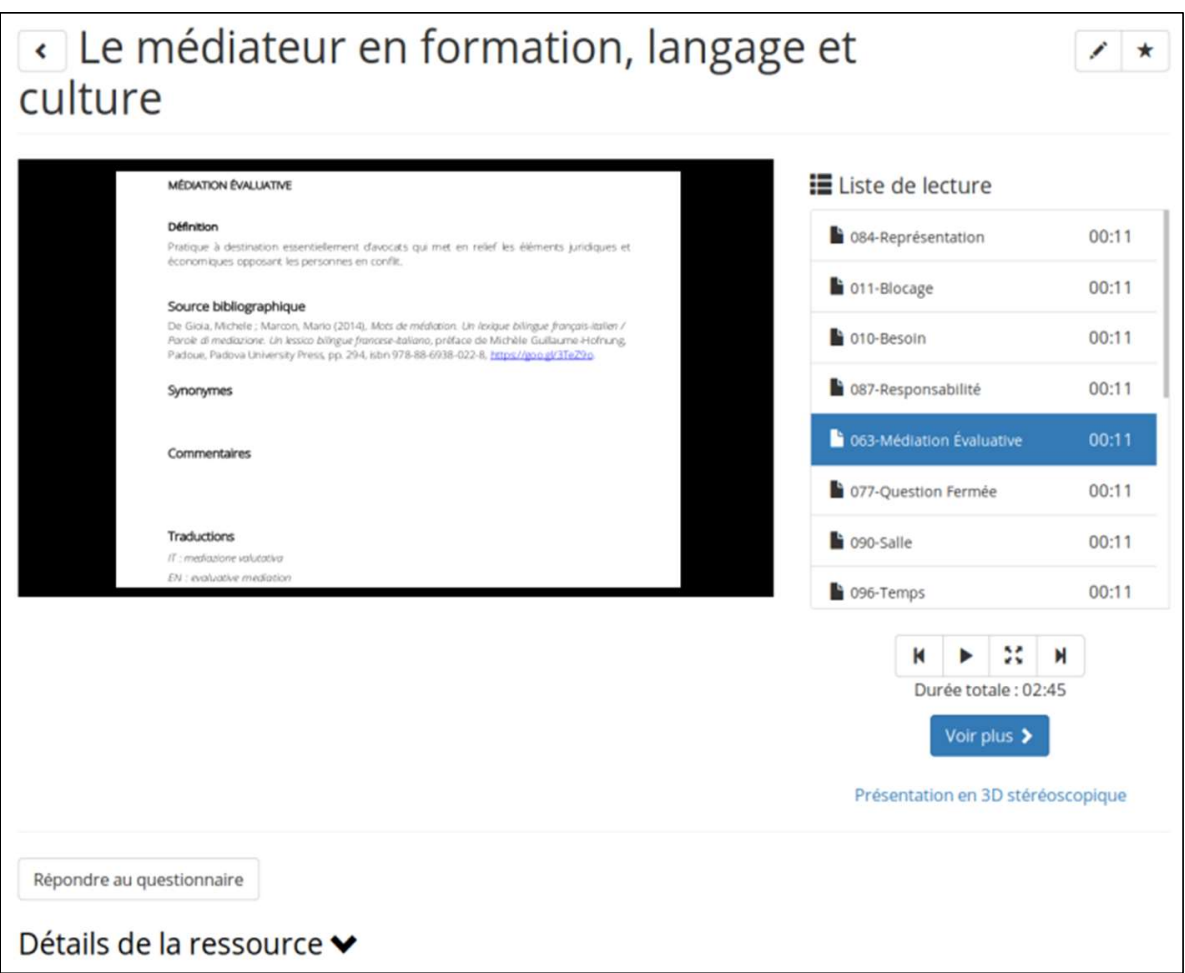

Figure 3.3.1-7: Aperçu d'une présentation. Ici, l'utilisateur du système est, par exemple, en train d'étudier la définition du terme « médiation évaluative » à travers trois domaines d'occurrence.

Bien entendu, en cas d'utilisation dans un contexte pédagogique, toutes les fonctions classiques d'un lecteur multimédia sont natives (i.e. elles sont disponibles sans sortir du système).

On vient de le voir, la création d'une présentation peut être dédoublée, avec un matériau sémantique que l'utilisateur peut juger important et/ou nécessaire (liste initiale) et un matériau secondaire, objet de compléments d'approfondissement (liste suggérée). À tout moment, on peut activer l'une ou l'autre, voire les deux. On accède à cette fonctionnalité en appuyant sur le bouton « Voir plus » de l'écran de la Figure précédente (3.3.1-7, en bas à droite). 


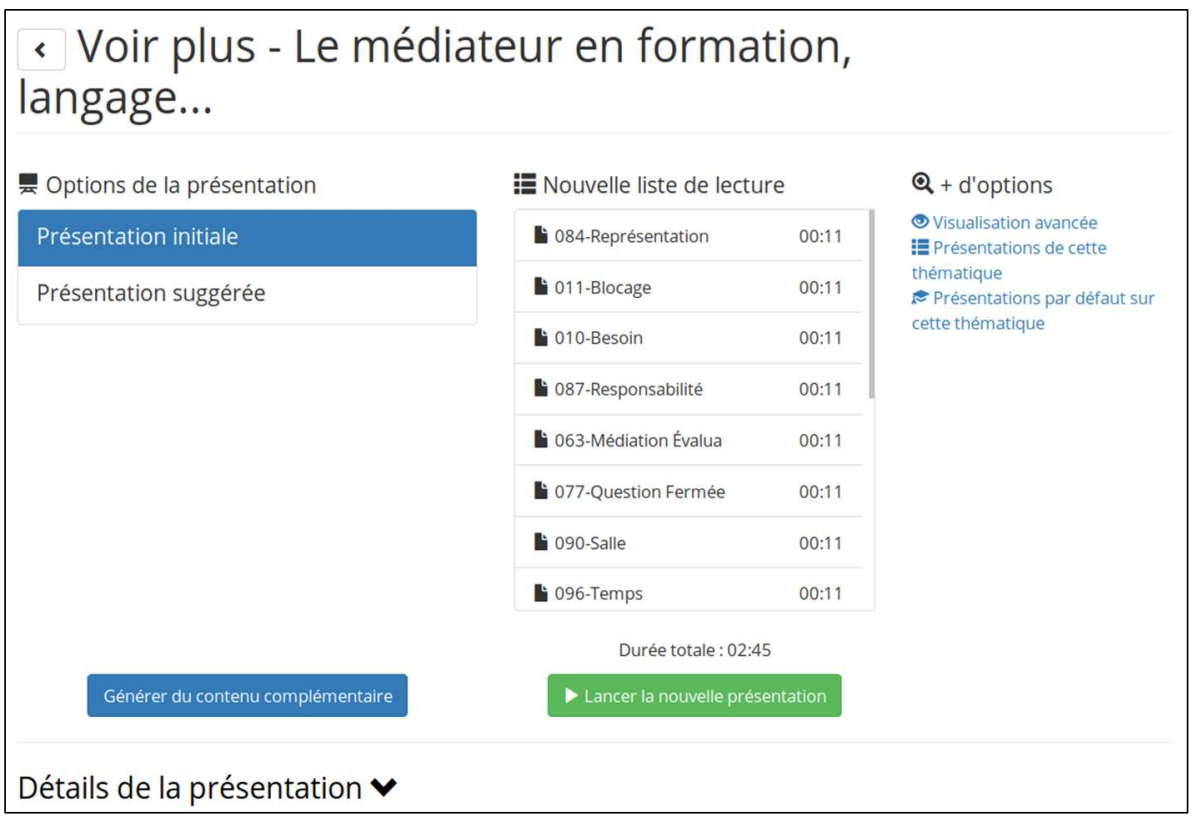

Figure 3.3.1-8 : L'interface illustre le moment où l'on cherche des compléments pouvant affiner la présentation initiale, qui contient le matériau jugé de base, (sélectionnée, en bleu).

Mais AMB intègre aussi une fonction « intelligente » qui permet d'affiner une présentation jusqu'à épuisement des ressources contenues dans la matrice sur laquelle se fonde la présentation. Effectivement, en appuyant sur "Générer du contenu complémentaire », $\mathrm{AMB}$ inspecte ce qui a déjà été présenté et, par un algorithme spécifiquement élaboré, en suivant une logique d'affinement incrémental, il propose des compléments, i.e., des ressources déjà contenues dans la matrice de la présentation, mais qui n'avaient été choisies dans aucune des deux listes de présentation (initiale et suggérée). Ce processus est récurrent et amène toujours du matériau terminologique complémentaire nouveau. Il ne s'arrête que lorsque toutes les ressources de la matrice ont été sélectionnées. Dans la figure suivante, on illustre le cas d'un complément demandé au système, faisant appel à la liste suggérée et à trois compléments. La nouvelle présentation contiendra, donc, outre la liste suggérée, trois autres compléments, chacun différent des autres ainsi que de la présentation de base (initiale) et de la présentation optionnelle (suggérée). Il est alors possible de progresser dans l'approfondissement de la sémantique d'un terme jusqu'au niveau de finesse sémantique souhaité. 


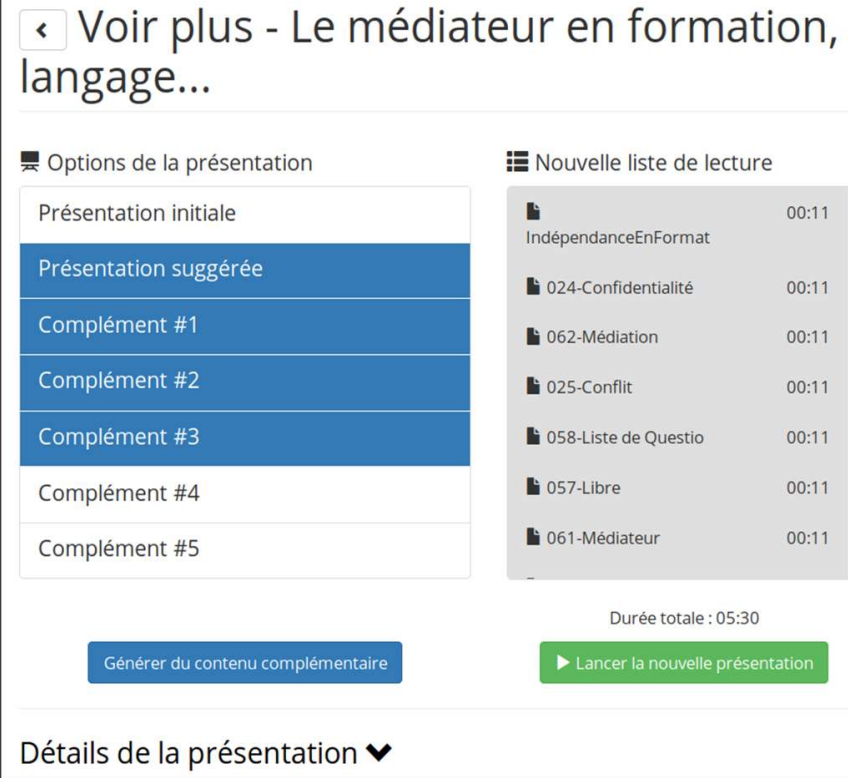

Détails de la présentation $\checkmark$

Figure 3.3.1.-9 : Interaction avec le système pour un affinement de la présentation initiale. Ici, on sollicite le système pour une présentation nouvelle faite à partir de la liste suggérée et des trois compléments proposés.

Le système $\mathrm{AMB}$ ne se limite pas à ces fonctionnalités. Mais celles que nous avons présentées nous semblent déjà suffisantes pour justifier son choix et assurer une prise en main efficace, dans le cadre du projet d'une « Onto-terminologie de la médiation ». Au moins, ce court descriptif a-t-il esquissé l'intérêt d'utiliser ce système et illustré son potentiel pour un usage plus exigeant dans la recherche terminologique. Une recherche qui ne se limiterait pas à une base de données, fûtelle étendue, faite de définitions et de références, visant la simple consultation des ressources textuelles, de façon isolée ; mais plutôt une recherche qui ferait appel à des syndications terminologiques au sein d'un discours explicatif ou argumentatif.

Terminons par une fonctionnalité à part, qui peut apporter, nous semble-t-il, une nouvelle perspective dans la notion même de « ressource terminologique ». Il s'agit de la possibilité d'annoter les ressources textuelles terminologiques.

\subsubsection{Annotation des ressources textuelles}

AMB dispose, en effet, d'un module permettant d'ajouter manuellement, et de façon qui ne demande pas un savoir-faire technique spécifique, des annotations textuelles ou même sonores, pendant l'étape de création d'une présentation. L'objectif est d'enrichir le contenu des ressources par des commentaires, fonctionnant comme des amendements positifs de nature inter-sémiotique. 


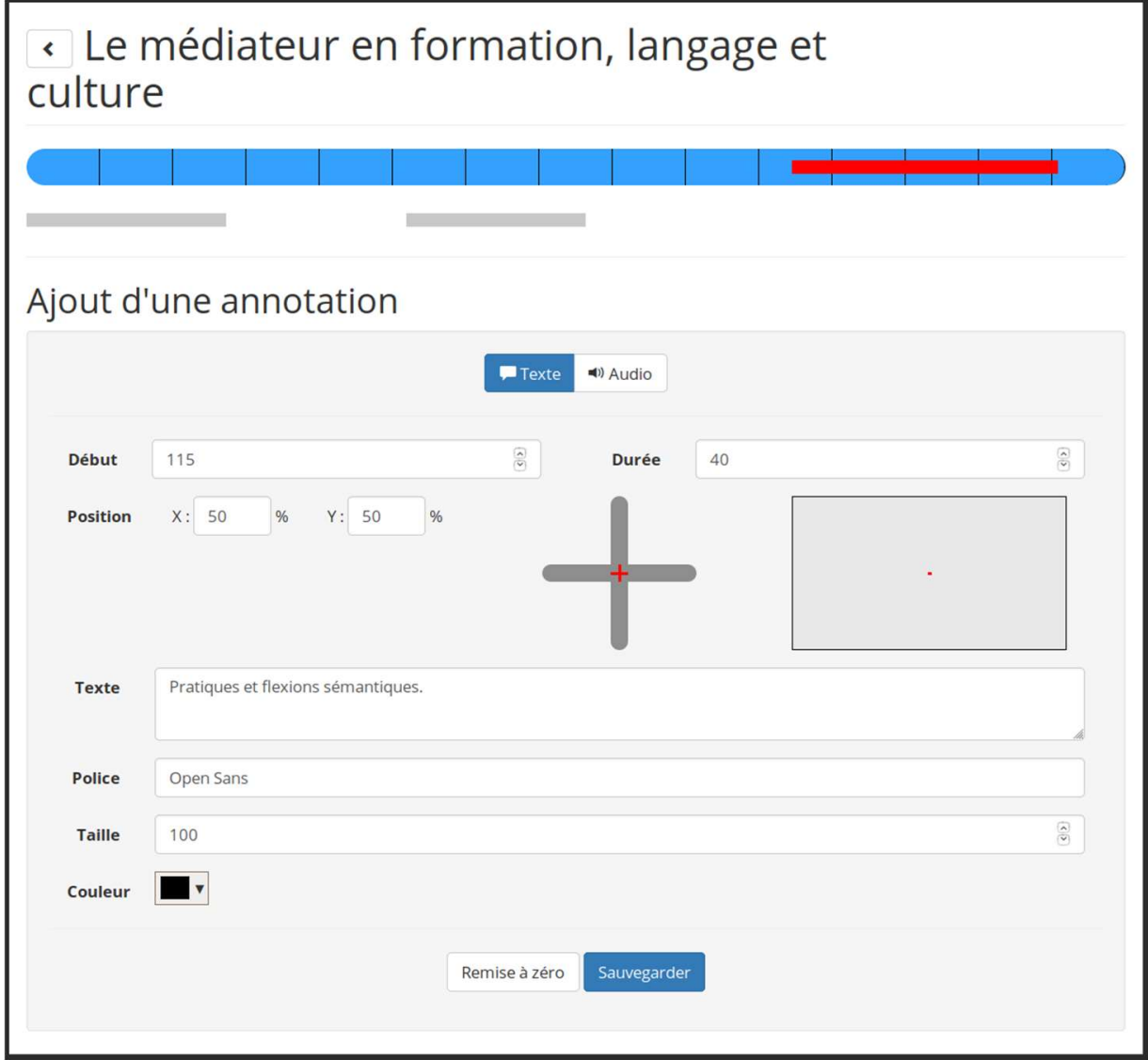

Figure 3.3.2 : Module d'annotation des présentations. La barre bleue tout en haut schématise les durées que les ressources occupent dans la présentation que nous avions créée (sur les variations du sens de " médiateur» dans trois domaines différents). Ici, on est en train de créer la troisième annotation (en rouge ; les deux autres sont figurées en gris, sous la barre bleue). On veut que cette annotation commence à la $115^{\mathrm{e}}$ seconde et qu'elle dure 40 secondes. On spécifie seulement son début et sa durée. On peut positionner la ressource où on veut spatialement sur une ressource (à n'importe quel endroit de la page) ; pour cela, des guides de position fournissent une triple aide. On peut aussi choisir la taille de l'annotation écrite, la police des caractères, etc. Mais une annotation terminologique peut aussi bien être sonore (un fichier audio ; par exemple, un commentaire enregistré pour une partie voire pour toute la présentation). Plusieurs annotations sont possibles, même simultanément. Annotations textuelles et sonores peuvent même coexister.

Cette fonctionnalité augure - du moins, nous le souhaitons - une terminologie enrichie ainsi que des moyens de médiation terminologique multimédia, configurables rapidement, sans grand coût de production. 


\section{Perspectives}

Deux mots de conclusion sur l'avenir du projet.

Le concept de médiation étant, d'évidence, destiné à une longue et riche carrière, notre projet ne serait pas à court d'inspiration ou d'intérêt. Son sort est cependant lié à deux autres facteurs, qui présentent des risques :

I. Le premier, mais qui est secondaire en importance, est l'avenir des technologies qui le supportent, et plus spécifiquement du système AMB. Dans une société numérique, qui est désormais la nôtre, ce risque est général et permanent. Les technologies, effectivement, se renouvellent à des rythmes rapides, rendant obsolètes les systèmes au bout de deux ou trois ans. Ce qui demande une remise à jour des systèmes suivant les nouvelles versions des langages, frameworks, etc., voire des normes sécuritaires. Il s'agit d'un risque impossible à éliminer mais parfaitement gérable, avec un budget maîtrisé.

II. Le second, et principal, concerne l'accueil qui peut être réservé à la méthodologie et plus généralement au paradigme de travail qu'il propose, au sein d'une communauté qui saurait le soutenir et l'enrichir, en s'appropriant sa philosophie et en le mettant à l'épreuve de nouveaux défis terminologiques. Ce risque est, hélas, imprévisible. Il ne dépend pas seulement de l'intérêt d'un système, d'un mode de travail ou d'une méthode. Il dépend d'une sorte de "marketing académique » rendant visible son intérêt, mais aussi des ressources que le projet pourrait trouver pour assurer sa continuité.

Dans tous les cas, notre projet ne visait, comme nous l'avons déjà expliqué, que de montrer son intérêt et la faisabilité de l'approche. Du point de vue de son contenu, son avenir immédiat consistera :

i. À le faire connaître par des voies traditionnelles de diffusion académique.

ii. À augmenter la base des ressources, tant en nombre des textes à impact terminologique pour les 7 termes de la matrice réduite proposée, que pour les autres 91 (98-7) termes de la liste initiale. Éventuellement aussi, à s'ouvrir à de nouveaux domaines.

iii. À imaginer de nouveaux usages d'utilisation du système AMB avec l'ontoterminologie, avec, chaque fois, l'implémentation de l'onto-terminologie, notamment des usages pédagogiques dans le domaine de la terminologie. AMB pouvant soutenir des paradigmes éducatifs relevant de la pédagogie active (classes inversées, pédagogies par projet, etc.), il serait tout à fait possible de faire travailler des étudiants, par exemple, sur des questions de terminologie particulières, dans le cadre de leur projet de formation, en utilisant la méthodologie proposée via le système AMB pour consigner le fruit de leur réflexion. 
À la fois ouvert à des opérations de recherche et de formation, nous imaginons, à court terme, de faire monter en qualité et en quantité, mais aussi en multipliant les usages, ces premiers acquis du projet.

\section{BIBLIOGRAPHIE}

ANDERSON Benedict (1983), Imagined Communities, London, Verso.

ANTIN Rica Simona, KANELlos Ioannis (2015), Un système de médiation pédagogique pour une assistance interprétative, in: Actes du VIIIe colloque QPES 2015: Questions de pédagogies dans l'enseignement supérieur Innover : pourquoi et comment? (Brest, 17-19 juin 2015), Brest, France, p. 702715, <https://goo.gl/qDKfAk> (dernier accès en mars 2019).

ANTIN Rica Simona, Bella Gábor, KANELlos Ioannis (2013), Un système d'exposition interactif pour une médiation scientifique pluri-lectoriale, in : Actes du VIle colloque QPES 2013: Questions de pédagogies dans l'enseignement supérieur - Les innovations pédagogiques en enseignement supérieur : pédagogies actives en présentiel et à distance (Sherbrooke, Canada, 3-5 juin 2013), p. 808-816, <https://goo.gl/yMqcGj> (dernier accès en mars 2019).

Antin Rica Simona, Kanellos Ioannis, Houssais Antoine, Transon Alan, De BOUgRenet DE LA TOCNAYE Jean-Louis, GRENIER Damien, CURT JeanBaptiste, Tritas Aris, CAROFF Anne-Gabrielle, MeILlerais Romain (2016), Cultural and educational mediation meets multimedia-based adaptive storytelling: A profile-sensitive system for personalized presentations, Mediterranean Archaeology and Archaeometry 16/5, p. 105-113, $<$ https://tinyurl.com/y2yo9rav $>$ (dernier accès en mars 2019).

BEN MRAD Fathi (2006), Équité, neutralité, responsabilité. À propos des principes de la médiation, Négociations $5 / 1$, p. 51-65, <https://tinyurl.com/y $5 \mathrm{hmf68v}>$ (dernier accès en mars 2019).

BEN MRAD Fathi (2018), Interactions communicatives en médiation, Paris, L'Harmattan.

Ben Mrad Fathi, Marchal Hervé, StéBÉ Jean-Marc (dir.) (2008), Penser la médiation, Paris, L'Harmattan.

Bhatia Vijay K., CANDlin Christopher N., GotTi Maurizio (éd.) (2010), The Discourses of Dispute Resolution, Berne, Peter Lang.

Bourigault Didier, SLODZIAN Monique (1999), Pour une terminologie textuelle, Terminologies nouvelles 19, p. 29-32.

BRUNI Alessandro, STIZIA Michela (2011), Mediazioni civili e commerciali. 30 casi di successo, Santarcangelo di Romagna, Maggioli.

CASTELli Stefano (1996), La mediazione. Teorie e Tecniche, Milan, Raffaello Cortina.

CAUNE Jean (1999), Pour une éthique de la médiation: le sens des pratiques culturelles, Grenoble, Pug. 
CAUNE Jean (2006), Culture et communication : convergences théoriques et lieux de médiation, Grenoble, Pug.

CAUne Jean (2013), Pour des humanités contemporaines. Science, technique, culture : quelles médiations?, Grenoble, Pug.

Coste Daniel, CAVALli Marisa (2015), Éducation, mobilité, altérité. Les fonctions de médiation de l'école, Unité des Politiques linguistiques - Division des politiques éducatives, Conseil de l'Europe, <https://tinyurl.com/yyr8k3tg> (dernier accès en mars 2019).

DE GIOIA Michele (dir.) (2013), Autour de la traduction juridique, Padoue, Padova University Press.

De GiOIA Michele (éd.) (2014), Pratiques communicatives de la médiation. Actes du Colloque international (Université de Padoue, 6-7 décembre 2012), Berne, Peter Lang.

DE GIOIA Michele (2019), La médiation et les sciences du langage à l'Université de Padoue (2010-2019), in : GuILlaUME-HofNuNG Michèle (éd.), L'impérieuse nécessité de la médiation, in : Envies d'[im]Pertinences, Paris, Académie de l'Éthique, p. 113-126.

De Giola Michele, MARCON Mario (2014), Mots de médiation. Un lexique bilingue français-italien / Parole di mediazione. Un lessico bilingue francese-italiano, préface de Michèle GuILLAUME-Hofnung, Padoue, Padova University Press.

DE GIOIA Michele, MARCON Mario (dir.) (2016), Approches linguistiques de la médiation, Limoges, Lambert-Lucas.

De Giola Michele, MARCon Mario (dir.) (2020), L'essentiel de la médiation. Le regard des sciences humaines et sociales, Bruxelles, Peter Lang.

De GiOIA Michele, Agresti Giovanni, MARCON Mario (coord.) (2016), Médiation et droits linguistiques. Actes du Colloque international (Université de Padoue, 23 janvier 2014), in : Études de Linguistique Appliquée 181, Paris, Klincksieck / Didier Érudition.

De GiOIA Michele, Gourvès-Hayward Alison, SABLÉ Cathy (éd.) (2017), Acteurs et formes de médiation pour le dialogue interculturel. GLAT Padova 2016. Actes $d u$ Colloque international (Université de Padoue, 17-19 mai 2016), Brest, Télécom Bretagne, Institut Mines-Télécom.

Delamotte Régine (2004), Les médiations langagières, vol. 1 : Des faits de langue aux discours, vol. 2 : Des discours aux acteurs sociaux, Rouen, Pur / Cnrs.

FELBER Helmut (1987 [1984]), Manuel de terminologie, Paris, Unesco / Infoterm, $<$ https://tinyurl.com/y28y2n22> (dernier accès en juin 2019).

FIORUCCI Massimiliano (2000), La mediazione culturale. Strategie per l'incontro, Rome, Armando.

GAUDIN François (2005), La socioterminologie, Langages 157, p. 80-92.

GAVIOLI Laura (éd.) (2009), La mediazione linguistico-culturale : una prospettiva interazionista, Perugia, Guerra.

GELLEREAU Michèle (2011), Les médiations de la visite guidée, in : MARGARITO Mariagrazia, HÉDIARD Marie, CELOTTI Nadine (éd.), La comunicazione turistica. Lingue, culture, istituzioni a confronto / La communication touristique. Langues, cultures, institutions en face à face, Turin, Cortina, p. 199209. 
Greco Morasso Sara (2011), Argumentation in Dispute Mediation. A Reasonable Way to Handle Conflict, Amsterdam/Philadelphia, John Benjamins.

GRUBER Thomas R. (1993), Toward Principles for the Design of Ontologies Used for Knowledge Sharing, Technical Report KSL 93-04, Knowledge Systems Laboratory, Stanford University, <https://tinyurl.com/yyaogpza $>$ (dernier accès en mars 2019).

GUARINO Nicola (1998), Formal Ontology and Information Systems, amended version of a paper appeared in: GUARINO Nicola (éd.), Formal Ontology in Information Systems. Proceedings of FOIS'98, Trento, Italy, 6-8 June 1998, Amsterdam, Ios, p. 3-15, <https://tinyurl.com/ufzo5n3> (dernier accès en mars 2019).

GuARINO Nicola, OBERLE Daniel, StAAB Steffen (2009), What Is an Ontology?, in: STAAB Steffen, STUDER Rudi (éd.), Handbook on Ontologies, Berlin/Heidelberg, Springer, p. 1-17, <https://tinyurl.com/y5grk2zg > (dernier accès en mars 2019).

Guillaume-HofNung Michèle (2002), Le concept de médiation et l'urgence théorique, Les Cahiers du CREMOC 35, p. 17-25.

Guillaume-HofNung Michèle (2015 [1995]), La médiation, $7^{\mathrm{e}}$ éd. mise à jour, Paris, Puf.

ISO 704 (2009), Travail terminologique - Principes et méthodes, $3^{\mathrm{e}}$ éd., Genève, Organisation Internationale de Normalisation.

KANELLOS Ioannis (2019), Lecture et relectures : sur une forme de symétrisation entre l'agir et le faire-agir interprétatifs, in: GROUPE $v$ (éd.), Bilan de l'intelligence. Quelles places pour les utilisateurs, les textes et les cultures dans les environnements numériques de travail?, Caen, Puc.

KISTER Laurence, JACQUEY Evelyne, GAIFFE Bertrand (2011), Du thesaurus à l'onto-terminologie: relations sémantiques vs relations ontologiques, Corela $9 / 1,<$ https://tinyurl.com/y2zwusj7 > (dernier accès en octobre 2020).

KLEIBER Georges (1991), La sémantique du Prototype, catégories et sens lexical, Paris, Puf.

L'Homme Marie-Claude (2004), La terminologie: principes et techniques, Montréal, Les Presses de l'Université de Montréal.

LAFORTUNE Jean-Marie (2012), La médiation culturelle. Le sens des mots et l'essence des pratiques, Quebec, Puq.

LAMIZET Bernard (1999), La médiation culturelle, Paris, L'Harmattan.

LASCOUX Jean-Louis (2001), Pratique de la médiation. Une méthode alternative à la résolution de conflits, Issy-les-Moulineaux, Esf.

Lempereur Alain Pekar, SAlzer Jacques, COLSON Aurélien (2008), Méthode de médiation. Au cœur de la conciliation, Paris, Dunod.

MERZEAU Louise (2012), La médiation identitaire, Revue Française des Sciences de l'information et de la communication 1, <https://goo.gl/isSnXa $>$ (dernier accès en mars 2019).

MEYER Ingrid, MACKINTOSH Kristen (2000), 'L'étirement' du sens terminologique : aperçu du phénomène de la déterminologisation, in: THOIRON Philippe, BÉJOINT Henri (éd.), Le sens en terminologie, Lyon, Pul, p. 198-217.

RASTIER François (2009), Sémantique interprétative, Paris, Puf. 
Ruscetta Felice, CARAdOnNa Marcella, Novelli Francesco (éd.) (2011), La mediazione civile, Milan, Ipsoa \& Wolters Kluwer.

SINCLAIR John (1991), Corpus, concordance, collocation, Oxford, Oxford University Press.

SIX Jean-François (2001 [1990]), Le temps des médiateurs, Paris, Seuil.

SIX Jean-François (2002), Les médiateurs, Paris, Le Cavalier Bleu.

SMETS-GARY Coralie, BECKER Martine (2012), Médiation et techniques de négociation intégrative. Approche pratique en matière civile, commerciale et sociale, Bruxelles, Larcier.

TEMMERMAN Rita (2000), Une théorie réaliste de la terminologie: le sociocognitivisme, Terminologies nouvelles 21, p. 58-64.

VÉZINA Robert, DARRAS Xavier, BÉDARD Jean, LAPOINTE-GIGUÈRE Micheline (2009), La rédaction des définitions terminologiques, Montréal, Office québécois de la langue française, <https://tinyurl.com/y4foy8mu $>$ (dernier accès en février 2019).

VIDAL Geneviève (2003), Interactivité et médiation dans l'usage des multimédias de musées, Communication \& Langage 137/1, p. 63-76.

Vocabulaire de la médiation professionnelle, <https://goo.g1/QWV41j> (dernier accès en mars 2019).

WÜSTER Eugen (1981), L'étude scientifique générale de la terminologie, zone frontalière entre la linguistique, la logique, l'ontologie, l'informatique et les sciences des choses, in : RONDEAU Guy, FELBER Helmut, SIFOROV Vladimir Ivanovich (éd.), Textes choisis de terminologie. I. Fondements théoriques de la terminologie, Laval, Groupe interdisciplinaire de recherche scientifique et appliquée en terminologie, Université de Laval, p. 57-114.

YARN Douglas H. (1999), Dictionary of Conflict Resolution, San Francisco, Jossey Bass. 